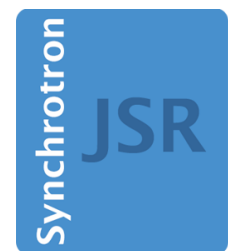

\title{
JOURNAL OF SYNCHROTRON RADIATION
}

Volume 22 (2015)

Supporting information for article:

High-accuracy X-ray absorption spectra from $\mathrm{m} M$ solutions of nickel (II) complexes with multiple solutions using transmission XAS

Christopher T. Chantler, M. Tauhidul Islam, Stephen P. Best, Lachlan J. Tantau, Chanh Q. Tran, Mun Hon Cheah and Andrew T. Payne 


\section{Supporting information - RV5033}

Table S1. Measured X-ray mass attenuation coefficients $\left[\begin{array}{c}\mu \\ \rho\end{array}\right]_{S}$ of the bis $(\mathrm{N}-\mathrm{i}-$ propyl-salicylaldiminato) nickel(II) complex from the intensity measurements $\left(I_{0}\right.$ and I) with a $15 \mathrm{mM}$ solution of the complex, and its corresponding solvent. Several experimental systematics including energy calibration, dark-current, solvent attenuations, harmonic contamination and thickness-ratio (from the actual solvent attenuation and fitted background of the sample (solution) were corrected to obtain the final result. The first column lists calibrated energies (Section 4.3) in $\mathrm{eV}$, and their associated uncertainties in parentheses. The second, third and fourth columns present corrected total X-ray mass attenuation coefficients $\left[\frac{\mu}{\rho}\right]_{S}$ with associated relative and percentage uncertainties. The fifth column lists the absolute uncertainties including the uncertainty contributions from the thickness ratio $t_{\text {ratio }}$ and column density $[\rho t]_{c}$ measurements. The sixth and the seventh columns list the (effective) photoelectric absorption coefficient $\left[\frac{\mu}{\rho}\right]_{p e}$ determined by subtracting the tabulated X-ray mass attenuations of the complex for the Rayleigh and Compton scattering from the total experimental X-ray mass attenuation coefficients $\left[\frac{\mu}{\rho}\right]_{S}$ at measured energies, with their corresponding uncertainties. The eighth column lists the X-ray mass attenuations of Rayleigh and Compton scattering, where the corresponding uncertainty of $\left[\frac{\mu}{\rho}\right]_{R+C}$ was determined from half of the variation between FFAST and XCOM tabulated values.

\begin{tabular}{|c|c|c|c|c|c|c|c|}
\hline $\begin{array}{c}\text { Energy } \\
(\mathrm{eV}) \\
\end{array}$ & $\begin{array}{c}{\left[\frac{\mu}{\rho}\right]_{s}} \\
\left(\mathrm{~cm}^{2} / g\right)\end{array}$ & $\begin{array}{c}\sigma_{\left[\frac{\mu}{\rho}\right]_{r e l}} \\
\left(\mathrm{~cm}^{2} / \mathrm{g}\right) \\
\end{array}$ & $\begin{array}{c}\sigma_{\left[\frac{\mu}{\rho}\right](r e l)} \\
(\%) \\
\end{array}$ & $\begin{array}{c}\sigma_{\left[\frac{\mu}{\rho}\right]_{a b s}} \\
\left(\mathrm{~cm}^{2} / \mathrm{g}\right) \\
\end{array}$ & $\begin{array}{c}{\left[\frac{\mu}{\rho}\right]_{p e}} \\
\left(\mathrm{~cm}^{2} / \mathrm{g}\right)\end{array}$ & $\begin{array}{c}\sigma_{\left[\frac{\mu}{\rho}\right]_{p e}} \\
\left(\mathrm{~cm}^{2} / g\right) \\
\end{array}$ & $\begin{array}{l}{\left[\frac{\mu}{\rho}\right]_{R+C}} \\
\left(\mathrm{~cm}^{2} / \mathrm{g}\right)\end{array}$ \\
\hline $7016.75(10)$ & 21.5226 & 0.6013 & 2.79 & 0.6562 & 20.8524 & 0.6014 & $0.670(11)$ \\
\hline $7618.05(08)$ & 14.8641 & 0.2674 & 1.80 & 0.3056 & 14.2368 & 0.2678 & $0.627(14)$ \\
\hline 8019.31(09) & 12.4312 & 0.2454 & 1.97 & 0.2774 & 11.8503 & 0.2455 & $0.581(08)$ \\
\hline 8019.36(12) & 13.4087 & 0.3431 & 2.56 & 0.3777 & 12.8278 & 0.3432 & $0.581(08)$ \\
\hline $8059.16(07)$ & 13.4538 & 0.1749 & 1.30 & 0.2096 & 12.8818 & 0.1756 & $0.572(15)$ \\
\hline $8099.42(07)$ & 12.7546 & 0.2021 & 1.58 & 0.2350 & 12.1916 & 0.2033 & $0.563(22)$ \\
\hline $8139.14(07)$ & 12.9023 & 0.1640 & 1.27 & 0.1972 & 12.3479 & 0.1665 & $0.554(29)$ \\
\hline $8179.60(07)$ & 10.7739 & 0.1630 & 1.51 & 0.1909 & 10.2290 & 0.1670 & $0.545(36)$ \\
\hline $8219.50(07)$ & 11.6627 & 0.1525 & 1.31 & 0.1826 & 11.1280 & 0.1591 & $0.535(45)$ \\
\hline $8259.75(08)$ & 11.6365 & 0.2348 & 2.02 & 0.2649 & 11.1113 & 0.2412 & $0.525(55)$ \\
\hline $8259.80(12)$ & 11.0898 & 0.2378 & 2.14 & 0.2665 & 10.5647 & 0.2441 & $0.525(55)$ \\
\hline 8299.69(07) & 12.1917 & 0.1382 & 1.13 & 0.1697 & 11.6760 & 0.1525 & $0.516(64)$ \\
\hline $8328.92(07)$ & 13.0854 & 0.1353 & 1.03 & 0.1690 & 12.5592 & 0.1456 & $0.526(54)$ \\
\hline $8329.93(07)$ & 13.1148 & 0.1287 & 0.98 & 0.1625 & 12.5849 & 0.1381 & $0.530(50)$ \\
\hline $8330.88(07)$ & 13.6350 & 0.1367 & 1.00 & 0.1718 & 13.1015 & 0.1444 & $0.534(47)$ \\
\hline $8331.95(07)$ & 13.4321 & 0.1420 & 1.06 & 0.1765 & 12.8946 & 0.1482 & $0.538(43)$ \\
\hline $8332.78(12)$ & 13.5470 & 0.2232 & 1.65 & 0.2580 & 13.0065 & 0.2267 & $0.541(40)$ \\
\hline $8332.84(08)$ & 13.6453 & 0.1582 & 1.16 & 0.1933 & 13.1045 & 0.1630 & $0.541(39)$ \\
\hline $8333.73(07)$ & 14.1161 & 0.1316 & 0.93 & 0.1679 & 13.5771 & 0.1352 & $0.539(31)$ \\
\hline $8334.75(07)$ & 14.5188 & 0.1273 & 0.88 & 0.1646 & 13.9762 & 0.1302 & $0.543(27)$ \\
\hline
\end{tabular}




\begin{tabular}{|c|c|c|c|c|c|c|c|}
\hline $8335.70(07)$ & 14.7894 & 0.1555 & 1.05 & 0.1935 & 14.2434 & 0.1574 & $0.546(24)$ \\
\hline $8336.65(07)$ & 14.7551 & 0.1332 & 0.90 & 0.1711 & 14.2058 & 0.1348 & $0.549(21)$ \\
\hline 8337.71(07) & 14.6010 & 0.1266 & 0.87 & 0.1641 & 14.0481 & 0.1277 & $0.553(17)$ \\
\hline $8338.72(07)$ & 15.3682 & 0.1310 & 0.85 & 0.1704 & 14.8119 & 0.1317 & $0.556(14)$ \\
\hline 8339.21(07) & 15.4175 & 0.1297 & 0.84 & 0.1692 & 14.8596 & 0.1303 & $558(12)$ \\
\hline 8339.63(07) & 15.7792 & 0.1323 & 0.84 & 0.1727 & 15.2200 & 0.1327 & $0.559(11)$ \\
\hline $8340.10(07)$ & 16.2828 & 0.1353 & 0.83 & 0.1770 & 15.7220 & 0.1356 & $0.561(09)$ \\
\hline 8340.64(07) & 17.2415 & 0.1331 & 0.77 & 0.1773 & 16.6791 & 0.1333 & $0.563(08)$ \\
\hline 8341.11(07) & 17.9227 & 0.1334 & 0.74 & 0.1792 & 17.3588 & 0.1335 & $0.564(06)$ \\
\hline $8341.65(07)$ & 19.4273 & .1291 & 0.66 & 0.1787 & 18.8618 & 0.1292 & $0.566(05)$ \\
\hline $8342.12(07)$ & 20.5521 & .1233 & 0.60 & 0.1758 & 19.9852 & 0.1234 & $567(03)$ \\
\hline $8342.60(07)$ & 21.9244 & .1209 & 0.55 & 0.1768 & 21.3561 & 0.1209 & $0.568(02)$ \\
\hline 8343.13(07) & 23.8207 & 0.1322 & 0.55 & 0.1928 & 23.2509 & 0.1322 & $0.570(00)$ \\
\hline 8343.73(07) & 26.0666 & .1338 & 0.51 & 0.2001 & 25.4951 & 1338 & $572(02)$ \\
\hline $8344.20(07)$ & 28.8136 & 1365 & 0.47 & 0.2098 & 28.2408 & 0.1366 & $0.573(03)$ \\
\hline $8344.69(08)$ & 32.4253 & 0.1787 & 0.55 & 0.2610 & 31.8512 & 0.1787 & $0.574(04)$ \\
\hline $8344.75(12)$ & 32.4296 & 0.2346 & 0.72 & 0.3169 & 31.8554 & 0.2346 & $0.574(04)$ \\
\hline 8345.23(07) & 35.3511 & 0.1262 & 0.36 & 0.2158 & 34.7757 & 0.1263 & $575(05)$ \\
\hline $8345.64(12)$ & 39.1722 & 2157 & 0.55 & 0.3149 & 38.5958 & 0.2158 & $576(06)$ \\
\hline $8345.70(08)$ & 39.1773 & 1620 & 0.41 & 0.2612 & 38.6007 & 0.1621 & $0.577(07)$ \\
\hline 8346.18(07) & 42.7013 & 0.1275 & 0.30 & 0.2356 & 42.1235 & 0.1277 & $0.578(08)$ \\
\hline 8346.71(07) & 46.9622 & 0.1311 & 0.28 & 0.2499 & 46.3833 & 0.1314 & $0.579(09)$ \\
\hline $8347.25(07)$ & 51.2888 & 0.1239 & 0.24 & 0.2536 & 50.7087 & 0.1244 & $0.580(10)$ \\
\hline $8347.72(07)$ & 56.6255 & 1597 & 0.28 & 0.3029 & 56.0444 & 0.1601 & $0.581(11)$ \\
\hline 8348.26(07) & 61.1106 & .1254 & 0.21 & 0.2798 & 60.5284 & 0.1260 & $0.582(12)$ \\
\hline $8348.80(07)$ & 65.9400 & 0.1305 & 0.20 & 0.2970 & 65.3568 & 0.1311 & $0.583(13)$ \\
\hline 8349.39(07) & 70.2153 & 0.1241 & 0.18 & 0.3013 & 69.6311 & 0.1249 & (14) \\
\hline 8349.88(07) & 73.7979 & 0.1271 & 0.17 & 0.3134 & 73.2128 & 0.1280 & $0.585(15)$ \\
\hline 8350.36(07) & 76.1117 & .1342 & 0.18 & 0.3263 & 75.5259 & 0.1352 & $0.586(16)$ \\
\hline $8350.83(07)$ & 77.8896 & 0.1229 & 0.16 & 0.3195 & 77.3032 & 0.1240 & $0.586(16)$ \\
\hline 8351.31(07) & 78.5225 & 0.1256 & 0.16 & 0.3238 & 77.9354 & 0.1268 & $0.587(17)$ \\
\hline 8351.85(07) & 78.9331 & 0.1277 & 0.16 & 0.3269 & 78.3455 & 0.1290 & $0.588(18)$ \\
\hline 8352.32(07) & 78.7748 & 0.1275 & 0.16 & 0.3263 & 78.1867 & 0.1288 & $0.588(18)$ \\
\hline 8352.92(07) & 79.0798 & 0.1280 & 0.16 & 0.3276 & 78.4911 & 0.1294 & $0.589(19)$ \\
\hline 8353.40(07) & 78.4030 & 0.1299 & 0.17 & 0.3278 & 77.8140 & 0.1313 & $0.589(19)$ \\
\hline 8353.81(08) & 77.4465 & 0.1663 & 0.21 & 0.3618 & 76.8572 & 0.1675 & $0.589(19)$ \\
\hline 8353.87(12) & 77.4726 & 0.2153 & 0.28 & 0.4108 & 76.8834 & 0.2162 & $0.589(19)$ \\
\hline 8354.35(11) & 76.8114 & 0.1996 & 0.26 & 0.3935 & 76.2219 & 0.2006 & $0.590(20)$ \\
\hline 8354.41(08) & 76.7963 & 0.1536 & 0.20 & 0.3474 & 76.2068 & 0.1549 & $0.590(20)$ \\
\hline 8354.96(07) & 75.8004 & 0.2431 & 0.32 & 0.4344 & 75.2107 & 0.2439 & $0.590(20)$ \\
\hline 8355.43(07) & 75.6457 & 0.1676 & 0.22 & 0.3585 & 75.0561 & 0.1687 & $0.590(20)$ \\
\hline 8355.91(11) & 73.8969 & 0.2174 & 0.29 & 0.4039 & 73.3073 & 0.2182 & $0.590(20)$ \\
\hline 8355.97(08) & 74.1280 & 0.1601 & 0.22 & 0.3472 & 73.5384 & 0.1613 & $0.590(20)$ \\
\hline $8356.45(07)$ & 73.0458 & 0.1318 & 0.18 & 0.3162 & 72.4564 & 0.1332 & $0.589(19)$ \\
\hline 8356.92(07) & 71.7103 & 0.1272 & 0.18 & 0.3082 & 71.1211 & 0.1286 & $0.589(19)$ \\
\hline 8357.40(07) & 70.4098 & 0.1397 & 0.20 & 0.3175 & 69.8210 & 0.1410 & $0.589(19$ \\
\hline
\end{tabular}




\begin{tabular}{|c|c|c|c|c|c|c|c|}
\hline 8357.82(11) & 69.6499 & 0.2141 & 0.31 & 0.3899 & 69.0614 & 0.2149 & $0.589(19)$ \\
\hline 8357.88(08) & 69.6967 & 0.1501 & 0.22 & 0.3261 & 69.1083 & 0.1513 & $0.589(18)$ \\
\hline 8358.36(07) & 68.4578 & 0.1323 & 0.19 & 0.3052 & 67.8698 & 0.1336 & $0.588(18)$ \\
\hline 8358.83(07) & 66.8694 & 0.1308 & 0.20 & 0.2997 & 66.2821 & 0.1320 & $0.587(17)$ \\
\hline 8359.37(07) & 66.4245 & .1245 & 0.19 & 0.2922 & 65.8379 & 0.1256 & $0.587(17)$ \\
\hline 8359.79(07) & 65.0605 & 0.1301 & 0.20 & 0.2944 & 64.4747 & 0.1311 & $0.586(16)$ \\
\hline $8360.27(07)$ & 64.0950 & 0.1381 & 0.22 & .3000 & 63.5101 & 0.1389 & $0.585(15)$ \\
\hline $8360.82(07)$ & 62.9721 & 0.2144 & 0.34 & 0.3735 & 62.3883 & 0.2149 & $0.584(14)$ \\
\hline $8361.35(07)$ & 62.9690 & 0.1239 & 0.20 & 0.2830 & 62.3865 & 0.1246 & $0.583(13)$ \\
\hline 8361.77(07) & 61.5139 & .1411 & 0.23 & 0.2965 & 60.9325 & 0.1415 & $0.581(11)$ \\
\hline $8362.25(07)$ & 60.7361 & 0.1252 & 0.21 & 0.2786 & 60.1561 & 0.1256 & $580(10)$ \\
\hline $8362.73(07)$ & 60.4462 & 0.1273 & 0.21 & .2800 & 59.8678 & 0.1276 & $0.579(08)$ \\
\hline 8363.21(07) & 59.8269 & 0.1398 & 0.23 & 0.2909 & 59.2501 & 0.1399 & 577(07) \\
\hline 8363.68(07) & 58.6372 & .1299 & 0.22 & 0.2781 & 58.0621 & 1300 & $575(05)$ \\
\hline 8364.10(07) & 57.9894 & .1467 & 0.25 & 0.2932 & 57.4160 & 0.1467 & $.573(03)$ \\
\hline $8364.58(07)$ & 58.0144 & 0.1262 & 0.22 & 0.2728 & 57.4430 & 0.1262 & $0.571(01)$ \\
\hline 8365.18(07) & 56.9563 & .1258 & 0.22 & .2697 & 56.3877 & 0.1258 & $.569(01)$ \\
\hline 8365.60(07) & 56.2500 & 0.1321 & 0.23 & 0.2742 & 55.6834 & 0.1321 & $567(03)$ \\
\hline $8366.09(07)$ & 55.2568 & 1351 & 0.24 & 0.2747 & 54.6927 & 0.1352 & $0.564(06)$ \\
\hline 8366.56(07) & 54.5053 & 1260 & 0.23 & 0.2638 & 53.9438 & 0.1263 & $0.562(09)$ \\
\hline 8367.16(07) & 53.8935 & 0.1323 & 0.25 & 0.2685 & 53.3355 & 0.1328 & $0.558(12)$ \\
\hline 8367.64(07) & 54.2388 & 0.1253 & 0.23 & 0.2624 & 53.6839 & 0.1262 & $0.555(15)$ \\
\hline 8368.12(07) & 53.2834 & 0.1233 & 0.23 & 0.2581 & 52.7316 & 0.1247 & $0.552(18)$ \\
\hline $8368.60(07)$ & 52.5589 & .1253 & 0.24 & 0.2582 & 52.0105 & 0.1271 & $0.548(22)$ \\
\hline 8369.02(07) & 51.9828 & 0.1300 & 0.25 & 0.2614 & 51.4375 & 0.1323 & $0.545(25)$ \\
\hline $8369.50(07)$ & 51.5661 & 0.1202 & 0.23 & 0.2506 & 51.0246 & 0.1235 & $0.542(29)$ \\
\hline 8370.03(07) & 51.0287 & 0.1381 & 0.27 & 0.2671 & 50.4915 & 0.1420 & $0.537(33)$ \\
\hline $8370.51(07)$ & 50.8573 & 0.1340 & 0.26 & 0.2626 & 50.3242 & 0.1390 & $0.533(37)$ \\
\hline $8371.00(07)$ & 49.8775 & 0.1400 & 0.28 & 0.2662 & 49.3488 & 0.1460 & $0.529(41)$ \\
\hline $8371.54(07)$ & 50.2645 & 0.1570 & 0.31 & 0.2841 & 49.7408 & 0.1637 & $0.524(46)$ \\
\hline $8372.02(07)$ & 49.3803 & 0.1256 & 0.25 & 0.2505 & 48.8614 & 0.1356 & $0.519(51)$ \\
\hline $8372.56(07)$ & 48.6748 & 0.1253 & 0.26 & 0.2484 & 48.1615 & 0.1376 & $0.513(57)$ \\
\hline 8373.10(07) & 48.3580 & 0.1224 & 0.25 & 0.2447 & 47.8507 & 0.1375 & $0.507(63)$ \\
\hline 8373.64(07) & 48.2735 & 0.1253 & 0.26 & 0.2474 & 47.7724 & 0.1430 & $0.501(69)$ \\
\hline 8374.12(07) & 47.9746 & 0.1281 & 0.27 & 0.2495 & 47.4793 & 0.1483 & $0.495(75)$ \\
\hline 8374.66(07) & 47.4471 & 0.1431 & 0.30 & 0.2632 & 46.9558 & 0.1634 & $0.491(79)$ \\
\hline $8375.14(07)$ & 47.2483 & 0.1242 & 0.26 & 0.2438 & 46.7563 & 0.1467 & $0.492(78)$ \\
\hline 8375.62(08) & 47.1789 & 0.2747 & 0.58 & 0.3941 & 46.6864 & 0.2855 & $0.493(78)$ \\
\hline 8376.10(07) & 47.9323 & 0.1291 & 0.27 & 0.2504 & 47.4391 & 0.1503 & $0.493(77)$ \\
\hline 8376.71(07) & 47.3021 & 0.1701 & 0.36 & 0.2898 & 46.8082 & 0.1864 & $0.494(76)$ \\
\hline $8377.25(07)$ & 47.5813 & 0.1632 & 0.34 & 0.2835 & 47.0868 & 0.1798 & $0.495(76)$ \\
\hline 8377.73(07) & 47.1579 & 0.1419 & 0.30 & 0.2612 & 46.6627 & 0.1605 & $0.495(75)$ \\
\hline $8378.27(07)$ & 47.4247 & 0.1345 & 0.28 & 0.2545 & 46.9289 & 0.1536 & $0.496(74)$ \\
\hline 8378.93(07) & 48.0327 & 0.2302 & 0.48 & 0.3517 & 47.5361 & 0.2416 & $0.497(73)$ \\
\hline 8380.91(07) & 49.5715 & 0.1263 & 0.25 & 0.2517 & 49.0724 & 0.1449 & $0.499(71)$ \\
\hline 8382.96(07) & 51.8672 & 0.1206 & 0.23 & 0.2517 & 51.3655 & 0.1386 & $0.502(68)$ \\
\hline
\end{tabular}




\begin{tabular}{|c|c|c|c|c|c|c|c|}
\hline $8385.06(07)$ & 54.2176 & 0.1216 & 0.22 & 0.2587 & 53.7133 & 0.1382 & $0.504(66)$ \\
\hline 8386.99(07) & 56.8360 & 0.1191 & 0.21 & 0.2627 & 56.3293 & 0.1349 & $0.507(63)$ \\
\hline 8388.98(07) & 57.8513 & 0.1290 & 0.22 & 0.2751 & 57.3422 & 0.1426 & $0.509(61)$ \\
\hline 8390.96(07) & 58.7225 & 0.1174 & 0.20 & 0.2658 & 58.2110 & 0.1311 & $0.512(58)$ \\
\hline 8392.90(07) & 59.2434 & 0.1589 & 0.27 & 0.3086 & 58.7295 & 0.1685 & $0.514(56)$ \\
\hline 8394.83(07) & 0.3381 & .1257 & 0.21 & 0.2782 & 59.8218 & 0.1367 & $0.516(54)$ \\
\hline $8396.75(07)$ & 60.6274 & 0.1353 & 0.22 & 0.2884 & 60.1087 & 0.1447 & $0.519(51)$ \\
\hline $3398.75(07)$ & 61.4334 & 0.1279 & 0.21 & 0.2831 & 60.9122 & 0.1369 & $0.521(49)$ \\
\hline $8400.74(07)$ & 61.3820 & 0.1273 & 0.21 & 0.2823 & 60.8584 & 0.1355 & $0.524(46)$ \\
\hline $8402.74(07)$ & 60.6579 & 0.1271 & 0.21 & 0.2803 & 0.1318 & 0.1345 & $0.526(44)$ \\
\hline $8404.80(07)$ & 61.2740 & 0.1117 & 0.18 & 0.2665 & 60.7454 & 0.1191 & $0.529(41)$ \\
\hline $8406.79(07)$ & 59.9347 & 0.1218 & 0.20 & 0.2733 & 59.4037 & 0.1279 & $0.531(39)$ \\
\hline 8408.98(07) & 59.3597 & 0.1250 & 0.21 & 0.2749 & 58.8260 & 0.1301 & $0.534(36)$ \\
\hline 8411.03(07) & 58.5838 & 0.1252 & 0.21 & 0.2732 & 58.0476 & 0.1297 & $.536(34)$ \\
\hline $8412.97(11)$ & 57.5036 & .1967 & 0.34 & 0.3420 & 56.9650 & 0.1992 & $0.539(32)$ \\
\hline 8413.04(08) & 57.5189 & .1510 & 0.26 & 0.2963 & 56.9803 & 0.1542 & $.539(31)$ \\
\hline 8415.03(07) & 57.5612 & 0.1255 & 0.22 & 0.2710 & 57.0202 & 0.1288 & $0.541(29)$ \\
\hline 8417.03(07) & 56.9572 & 0.1191 & 0.21 & 0.2630 & 56.4137 & 0.1220 & $0.544(27)$ \\
\hline $8418.98(07)$ & 56.1318 & 0.1289 & 0.23 & 0.2707 & 55.5860 & 0.1311 & $0.546(24)$ \\
\hline $8420.98(07)$ & 56.0614 & 1135 & 0.20 & 0.2552 & 55.5132 & 0.1155 & $0.548(22)$ \\
\hline 8422.92(07) & 56.3283 & .1168 & 0.21 & 0.2592 & 55.7777 & 0.1184 & $0.551(20)$ \\
\hline $8424.75(07)$ & 55.5757 & 0.1189 & 0.21 & 0.2594 & 55.0229 & 0.1201 & $0.553(17)$ \\
\hline $8426.75(07)$ & 55.3760 & 0.1202 & 0.22 & 0.2602 & 54.8208 & 0.1211 & $0.555(15)$ \\
\hline $8428.76(07)$ & 56.0353 & 0.1148 & 0.20 & 0.2564 & 55.4777 & 0.1155 & $0.558(12)$ \\
\hline $8430.65(07)$ & 55.3673 & .1159 & 0.21 & 0.2558 & 54.8075 & 0.1163 & $0.560(10)$ \\
\hline 8432.66(07) & 55.1509 & 0.1170 & 0.21 & 0.2564 & 54.5886 & 0.1173 & $0.562(08)$ \\
\hline $8434.80(07)$ & 54.3973 & 0.1199 & 0.22 & 0.2574 & 53.8325 & 0.1200 & $0.565(05)$ \\
\hline $8436.74(07)$ & 54.3083 & 0.1187 & 0.22 & 0.2560 & 53.7412 & 0.1187 & $0.567(03)$ \\
\hline $8438.81(07)$ & 54.5605 & 0.1201 & 0.22 & 0.2581 & 53.9908 & 0.1201 & $0.570(00)$ \\
\hline $8440.95(07)$ & 54.9725 & 0.1261 & 0.23 & 0.2651 & 54.4004 & 0.1262 & $0.572(02)$ \\
\hline 8442.90(07) & 54.7256 & 0.1166 & 0.21 & 0.2549 & 54.1512 & 0.1167 & $0.575(04)$ \\
\hline 8444.99(07) & 54.9653 & 0.1142 & 0.21 & 0.2532 & 54.3884 & 0.1145 & $0.577(07)$ \\
\hline 8447.06(07) & 55.1362 & 0.1182 & 0.21 & 0.2575 & 54.5568 & 0.1185 & $0.579(09)$ \\
\hline $8448.89(07)$ & 55.2674 & 0.1257 & 0.23 & 0.2654 & 54.6871 & 0.1261 & $0.580(10)$ \\
\hline $8450.85(11)$ & 55.4336 & 0.1906 & 0.34 & 0.3307 & 54.8536 & 0.1908 & $0.580(10)$ \\
\hline $8450.92(08)$ & 55.8605 & 0.2589 & 0.46 & 0.4001 & 55.2805 & 0.2591 & $0.580(10)$ \\
\hline 8452.93(07) & 55.6479 & 0.1202 & 0.22 & 0.2609 & 55.0681 & 0.1206 & $0.580(10)$ \\
\hline 8454.76(07) & 56.7590 & 0.1196 & 0.21 & 0.2630 & 56.1795 & 0.1199 & $0.580(09)$ \\
\hline $8456.67(07)$ & 56.1731 & 0.1213 & 0.22 & 0.2632 & 55.5938 & 0.1216 & $0.579(09)$ \\
\hline $8458.75(07)$ & 56.1686 & 0.1222 & 0.22 & 0.2642 & 55.5896 & 0.1225 & $0.579(09)$ \\
\hline $8460.65(07)$ & 56.3052 & 0.1147 & 0.20 & 0.2570 & 55.7265 & 0.1150 & $0.579(09)$ \\
\hline $8462.68(07)$ & 56.3645 & 0.1211 & 0.21 & 0.2636 & 55.7860 & 0.1214 & $0.579(08)$ \\
\hline 8464.82(07) & 56.4501 & 0.1173 & 0.21 & 0.2599 & 55.8719 & 0.1176 & $0.578(08)$ \\
\hline $8466.79(07)$ & 56.6298 & 0.1155 & 0.20 & 0.2586 & 56.0518 & 0.1158 & $0.578(08)$ \\
\hline $8468.88(07)$ & 56.5766 & 0.1207 & 0.21 & 0.2636 & 55.9990 & 0.1209 & $0.578(08)$ \\
\hline 8470.96(07) & 56.4156 & 0.1154 & 0.20 & 0.2580 & 55.8382 & 0.1156 & $0.577(07)$ \\
\hline
\end{tabular}




\begin{tabular}{|c|c|c|c|c|c|c|c|}
\hline $8472.94(07)$ & 56.3089 & 0.1176 & 0.21 & 0.2599 & 55.7318 & 0.1178 & $0.577(07)$ \\
\hline $8475.02(07)$ & 56.2737 & 0.1097 & 0.19 & 0.2519 & 55.6968 & 0.1099 & $0.577(07)$ \\
\hline $8477.06(07)$ & 56.4859 & 0.1133 & 0.20 & 0.2561 & 55.9093 & 0.1135 & $0.577(07)$ \\
\hline 8479.03(07) & 56.4396 & 0.1141 & 0.20 & 0.2568 & 55.8632 & 0.1143 & $0.576(06)$ \\
\hline $8481.00(07)$ & 56.6572 & 0.1147 & 0.20 & 0.2579 & 56.0811 & 0.1149 & $0.576(06)$ \\
\hline $8482.98(11)$ & 56.8976 & 0.2103 & 0.37 & 0.3541 & 56.3218 & 0.2104 & $0.576(06)$ \\
\hline $8483.04(08)$ & 56.7863 & 0.1437 & 0.25 & 0.2872 & 56.2104 & 0.1438 & $0.576(06)$ \\
\hline $8484.88(07)$ & 56.9381 & 0.1164 & 0.20 & 0.2603 & 56.3625 & 0.1166 & $0.576(06)$ \\
\hline $8486.79(07)$ & 56.9355 & 0.1206 & 0.21 & 0.2645 & 56.3601 & 0.1207 & $0.575(05)$ \\
\hline $8488.84(07)$ & 57.1642 & 0.1096 & 0.19 & 0.2541 & 56.5891 & 0.1097 & $0.575(05)$ \\
\hline $8490.81(07)$ & 57.0105 & 0.1163 & 0.20 & 0.2604 & 56.4356 & 0.1164 & $0.575(05)$ \\
\hline $8492.73(07)$ & 57.0065 & 0.1150 & 0.20 & 0.2590 & 56.4318 & 0.1151 & $0.575(05)$ \\
\hline $8494.77(07)$ & 57.0045 & 0.1116 & 0.20 & 0.2557 & 56.4301 & 0.1117 & $0.574(04)$ \\
\hline $8496.81(07)$ & 56.7802 & 0.1155 & 0.20 & 0.2590 & 56.2061 & 0.1156 & $0.574(04)$ \\
\hline $8498.85(07)$ & 56.7953 & 0.1204 & 0.21 & 0.2639 & 56.2215 & 0.1205 & $0.574(04)$ \\
\hline $8500.95(07)$ & 56.4702 & .1094 & 0.19 & 0.2521 & 55.8965 & 0.1095 & $0.574(04)$ \\
\hline $8503.07(07)$ & 56.2627 & 0.1143 & 0.20 & 0.2565 & 55.6891 & 0.1143 & $0.574(04)$ \\
\hline $8505.11(07)$ & 55.9909 & 0.1106 & 0.20 & 0.2521 & 55.4173 & 0.1107 & $0.574(04)$ \\
\hline $8507.15(07)$ & 55.6440 & 0.1135 & 0.20 & 0.2542 & 55.0705 & 0.1136 & $0.574(04)$ \\
\hline $8509.14(07)$ & 55.4463 & 0.1112 & 0.20 & 0.2513 & 54.8730 & 0.1112 & $0.573(04)$ \\
\hline $8511.06(07)$ & 55.1728 & .1056 & 0.19 & 0.2450 & 54.5995 & 0.1056 & $0.573(04)$ \\
\hline $8513.05(07)$ & 55.2909 & 0.3193 & 0.58 & 0.4591 & 54.7176 & 0.3193 & $0.573(04)$ \\
\hline $8515.04(07)$ & 55.1563 & 0.1192 & 0.22 & 0.2586 & 54.5832 & 0.1192 & $0.573(04)$ \\
\hline $8516.84(07)$ & 54.6673 & 0.1125 & 0.21 & 0.2507 & 54.0942 & 0.1126 & $0.573(04)$ \\
\hline $8519.08(05)$ & 54.2035 & 0.0840 & 0.15 & 0.2210 & 53.6305 & 0.0841 & $0.573(04)$ \\
\hline $8529.10(07)$ & 53.2853 & 0.1096 & 0.21 & 0.2443 & 52.7127 & 0.1097 & $0.573(04)$ \\
\hline $8539.59(07)$ & 53.5336 & 0.1164 & 0.22 & 0.2518 & 52.9615 & 0.1165 & $0.572(04)$ \\
\hline $8549.29(07)$ & 53.5485 & 0.1125 & 0.21 & 0.2478 & 52.9767 & 0.1126 & $0.572(05)$ \\
\hline $8559.27(07)$ & 53.4359 & 0.1210 & 0.23 & 0.2560 & 52.8645 & 0.1210 & $0.571(05)$ \\
\hline $8569.58(07)$ & 53.7493 & 0.1153 & 0.21 & 0.2512 & 53.1783 & 0.1154 & $0.571(05)$ \\
\hline $8579.29(07)$ & 53.1981 & 0.1147 & 0.22 & 0.2492 & 52.6275 & 0.1148 & $0.571(05)$ \\
\hline $8589.34(07)$ & 53.0783 & 0.1230 & 0.23 & 0.2572 & 52.5081 & 0.1231 & $0.570(05)$ \\
\hline $8599.66(05)$ & 52.3641 & 0.1045 & 0.20 & 0.2369 & 51.7944 & 0.1046 & $0.570(06)$ \\
\hline $8609.57(07)$ & 51.9303 & 0.1190 & 0.23 & 0.2503 & 51.3610 & 0.1191 & $0.569(06)$ \\
\hline $8619.50(11)$ & 52.0192 & 0.2446 & 0.47 & 0.3761 & 51.4503 & 0.2446 & $0.569(06)$ \\
\hline $8639.86(07)$ & 51.2346 & 0.1095 & 0.21 & 0.2391 & 50.6666 & 0.1097 & $0.568(06)$ \\
\hline $8659.76(07)$ & 50.0725 & 0.1152 & 0.23 & 0.2418 & 49.5053 & 0.1153 & $0.567(06)$ \\
\hline $8679.56(07)$ & 50.3457 & 0.1106 & 0.22 & 0.2380 & 49.7794 & 0.1108 & $0.566(07)$ \\
\hline $8699.91(07)$ & 49.5447 & 0.1132 & 0.23 & 0.2385 & 48.9793 & 0.1134 & $0.565(07)$ \\
\hline $8719.62(07)$ & 50.0301 & 0.1177 & 0.24 & 0.2442 & 49.4655 & 0.1179 & $0.565(07)$ \\
\hline $8739.71(07)$ & 48.7851 & 0.1292 & 0.26 & 0.2526 & 48.2215 & 0.1295 & $0.564(08)$ \\
\hline $8759.75(07)$ & 48.4790 & 0.1124 & 0.23 & 0.2351 & 47.9163 & 0.1127 & $0.563(08)$ \\
\hline $8779.56(05)$ & 48.0208 & 0.0903 & 0.19 & 0.2118 & 47.4590 & 0.0907 & $0.562(08)$ \\
\hline 8799.99(07) & 48.1171 & 0.1860 & 0.39 & 0.3077 & 47.5563 & 0.1862 & $0.561(09)$ \\
\hline $8819.84(07)$ & 47.2798 & 0.1134 & 0.24 & 0.2330 & 46.7199 & 0.1137 & $0.560(09)$ \\
\hline $8840.06(07)$ & 47.7177 & 0.1169 & 0.24 & 0.2376 & 47.1588 & 0.1172 & $0.559(09)$ \\
\hline
\end{tabular}




\begin{tabular}{llllllll}
$8870.37(07)$ & 46.9692 & 0.1497 & 0.32 & 0.2686 & 46.4116 & 0.1500 & $0.558(10)$ \\
$8900.29(07)$ & 47.0043 & 0.1167 & 0.25 & 0.2356 & 46.4482 & 0.1171 & $0.556(10)$ \\
$8930.46(05)$ & 46.9049 & 0.0853 & 0.18 & 0.2040 & 46.3503 & 0.0859 & $0.555(10)$ \\
$8960.45(08)$ & 46.0336 & 0.1406 & 0.31 & 0.2571 & 45.4805 & 0.1410 & $0.553(11)$ \\
$8960.52(12)$ & 46.1995 & 0.2094 & 0.45 & 0.3263 & 45.6464 & 0.2097 & $0.553(11)$ \\
$8990.29(07)$ & 46.1639 & 0.1181 & 0.26 & 0.2349 & 45.6123 & 0.1186 & $0.552(11)$ \\
$9020.47(07)$ & 46.1727 & 0.1197 & 0.26 & 0.2365 & 45.6225 & 0.1202 & $0.550(11)$ \\
$9060.67(07)$ & 45.8527 & 0.1148 & 0.25 & 0.2308 & 45.3041 & 0.1153 & $0.549(11)$ \\
$9100.96(12)$ & 45.3693 & 0.2017 & 0.44 & 0.3165 & 44.8224 & 0.2020 & $0.547(11)$ \\
$9141.02(07)$ & 44.1517 & 0.1169 & 0.26 & 0.2287 & 43.6066 & 0.1175 & $0.545(12)$ \\
$9181.25(09)$ & 43.5637 & 0.1455 & 0.33 & 0.2558 & 43.0203 & 0.1460 & $0.543(12)$ \\
$9181.32(12)$ & 43.3644 & 0.2063 & 0.48 & 0.3161 & 42.8210 & 0.2066 & $0.543(12)$ \\
$9221.31(06)$ & 43.4478 & 0.0930 & 0.21 & 0.2030 & 42.9062 & 0.0937 & $0.542(12)$ \\
$9261.30(05)$ & 42.9518 & 0.2084 & 0.49 & 0.3172 & 42.4120 & 0.2088 & $0.540(12)$ \\
$9301.21(07)$ & 43.2680 & 0.1242 & 0.29 & 0.2337 & 42.7300 & 0.1248 & $0.538(12)$ \\
$9341.23(09)$ & 42.6075 & 0.1549 & 0.36 & 0.2627 & 42.0714 & 0.1553 & $0.536(12)$ \\
$9341.30(13)$ & 42.3412 & 0.2201 & 0.52 & 0.3273 & 41.8051 & 0.2204 & $0.536(12)$ \\
$9381.16(08)$ & 42.7505 & 0.1298 & 0.30 & 0.2381 & 42.2163 & 0.1304 & $0.534(12)$ \\
$9421.50(08)$ & 42.4175 & 0.1344 & 0.32 & 0.2418 & 41.8851 & 0.1350 & $0.532(12)$ \\
$9471.79(14)$ & 42.8257 & 0.2412 & 0.56 & 0.3496 & 42.2957 & 0.2415 & $0.530(12)$ \\
$9471.87(10)$ & 42.8325 & 0.1709 & 0.40 & 0.2794 & 42.3026 & 0.1713 & $0.530(12)$ \\
$9522.39(08)$ & 42.8316 & 0.1457 & 0.34 & 0.2542 & 42.3041 & 0.1463 & $0.528(12)$ \\
\hline
\end{tabular}


Table S2. Measured X-ray mass attenuation coefficients $\left[{ }_{\rho}^{\mu}\right]_{S}$ of the bis $(\mathrm{N}-\mathrm{i}-$ propyl-salicylaldiminato) nickel(II) complex from the intensity measurements $\left(I_{0}\right.$ and $I$ ) with a $1.5 \mathrm{mM}$ solution of the complex, and its corresponding solvent. A number of experimental systematics including energy calibration, dark-current, solvent attenuations, harmonic contamination and thickness-ratio (from the actual solvent attenuation and fitted background of the sample (solution) were corrected to obtain the final result. The first column lists calibrated energies (Section 4.3) in $\mathrm{eV}$, and their associated uncertainties in parentheses. The second, third and fourth columns present corrected total X-ray mass attenuation coefficients $\left[\frac{\mu}{\rho}\right]_{S}$ with associated relative and percentage uncertainties. The fifth column lists the absolute uncertainties including the uncertainty contributions from the thickness ratio $t_{\text {ratio }}$ and column density $[\rho t]_{c}$ measurements. The sixth and the seventh columns list the (effective) photoelectric absorption coefficient $\left[\frac{\mu}{\rho}\right]_{p e}$ determined by subtracting the tabulated X-ray mass attenuations of the complex for the Rayleigh and Compton scattering from the total experimental X-ray mass attenuation coefficients $\left[\frac{\mu}{\rho}\right]_{S}$ at measured energies, with their corresponding uncertainties. The eighth column lists the X-ray mass attenuations of Rayleigh and Compton scattering, where the corresponding uncertainty of $\left[\frac{\mu}{\rho}\right]_{R+C}$ was determined from half of the variation between FFAST and XCOM tabulated values.

\begin{tabular}{|c|c|c|c|c|c|c|c|}
\hline $\begin{array}{c}\text { Energy } \\
(\mathrm{eV})\end{array}$ & $\begin{array}{c}{\left[\frac{\mu}{\rho}\right]_{s}} \\
\left(\mathrm{~cm}^{2} / g\right)\end{array}$ & $\begin{array}{c}\sigma_{\left[\frac{\mu}{\rho}\right]_{r e l}} \\
\left(\mathrm{~cm}^{2} / g\right)\end{array}$ & $\begin{array}{c}\sigma_{\left[\frac{\mu}{\rho}\right](r e l)} \\
(\%)\end{array}$ & $\begin{array}{c}\sigma_{\left[\frac{\mu}{\rho}\right]_{a b s}} \\
\left(\mathrm{~cm}^{2} / g\right)\end{array}$ & $\begin{array}{c}{\left[\frac{\mu}{\rho}\right]_{p e}} \\
\left(\mathrm{~cm}^{2} / g\right)\end{array}$ & $\begin{array}{c}\sigma_{\left[\frac{\mu}{\rho}\right]_{p e}} \\
\left(\mathrm{~cm}^{2} / \mathrm{g}\right)\end{array}$ & $\begin{array}{l}{\left[\frac{\mu}{\rho}\right]_{R+C}} \\
\left(\mathrm{~cm}^{2} / \mathrm{g}\right)\end{array}$ \\
\hline $8099.36(07)$ & 36.8201 & 1.6698 & 4.53 & 1.7699 & 36.2571 & 1.6699 & $0.563(22)$ \\
\hline $8139.20(07)$ & 24.6351 & 2.8668 & 11.6 & 2.9351 & 24.0807 & 2.8669 & $0.554(29)$ \\
\hline $8179.54(07)$ & 26.3899 & 1.4862 & 5.63 & 1.5592 & 25.8450 & 1.4867 & $0.545(36)$ \\
\hline $8219.56(07)$ & 14.2868 & 2.0371 & 14.2 & 2.0786 & 13.7521 & 2.0376 & $0.535(45)$ \\
\hline $8259.68(07)$ & 23.1324 & 1.3674 & 5.91 & 1.4319 & 22.6072 & 1.3685 & $0.525(55)$ \\
\hline $8299.75(07)$ & 13.4084 & 1.6295 & 12.2 & 1.6687 & 12.8928 & 1.6308 & $0.516(64)$ \\
\hline $8328.92(07)$ & 18.7673 & 1.2302 & 6.56 & 1.2833 & 18.2411 & 1.2314 & $0.526(54)$ \\
\hline 8329.93(07) & 20.3898 & 1.2591 & 6.18 & 1.3165 & 19.8599 & 1.2601 & $0.530(50)$ \\
\hline $8330.88(07)$ & 19.2183 & 1.2664 & 6.59 & 1.3207 & 18.6848 & 1.2672 & $0.533(47)$ \\
\hline 8331.95(07) & 21.2500 & 1.1905 & 5.60 & 1.2501 & 20.7125 & 1.1913 & $0.537(43)$ \\
\hline $8332.84(07)$ & 18.2139 & 1.2140 & 6.67 & 1.2657 & 17.6732 & 1.2146 & $0.541(39)$ \\
\hline $8333.73(07)$ & 21.3961 & 1.3173 & 6.16 & 1.3772 & 20.8571 & 1.3176 & $0.539(31)$ \\
\hline $8334.75(07)$ & 21.0412 & 1.2631 & 6.00 & 1.3222 & 20.4986 & 1.2634 & $0.543(27)$ \\
\hline $8335.70(07)$ & 22.5559 & 1.2547 & 5.56 & 1.3177 & 22.0099 & 1.2550 & $0.546(24)$ \\
\hline $8336.70(07)$ & 23.2202 & 1.2484 & 5.38 & 1.3131 & 22.6707 & 1.2486 & $0.549(21)$ \\
\hline $8337.71(07)$ & 22.0860 & 1.2646 & 5.73 & 1.3264 & 21.5331 & 1.2647 & $0.553(17)$ \\
\hline 8338.72(07) & 21.0854 & 1.2806 & 6.07 & 1.3397 & 20.5291 & 1.2806 & $0.556(14)$ \\
\hline $8339.21(07)$ & 22.1303 & 1.2761 & 5.77 & 1.3380 & 21.5725 & 1.2762 & $0.558(12)$ \\
\hline 8339.63(07) & 21.7090 & 1.3003 & 5.99 & 1.3610 & 21.1497 & 1.3003 & $0.559(11)$ \\
\hline $8340.10(07)$ & 23.6257 & 1.2924 & 5.47 & 1.3582 & 23.0649 & 1.2924 & $0.561(09)$ \\
\hline $8340.64(07)$ & 25.2605 & 1.2718 & 5.03 & 1.3418 & 24.6980 & 1.2718 & $0.562(08)$ \\
\hline 8341.11(07) & 26.6212 & 1.3845 & 5.20 & 1.4580 & 26.0573 & 1.3845 & $0.564(06)$ \\
\hline $8341.65(07)$ & 26.5812 & 1.2639 & 4.75 & 1.3373 & 26.0157 & 1.2639 & $0.566(04)$ \\
\hline $8342.12(07)$ & 29.5081 & 1.2332 & 4.18 & 1.3143 & 28.9412 & 1.2332 & $0.567(03)$ \\
\hline $8342.60(07)$ & 29.6686 & 1.2208 & 4.11 & 1.3022 & 29.1002 & 1.2208 & $0.568(02)$ \\
\hline
\end{tabular}




\begin{tabular}{|c|c|c|c|c|c|c|c|}
\hline $8343.13(07)$ & 32.5808 & 1.2454 & 3.82 & 1.3344 & 32.0109 & 1.2454 & $0.570(00)$ \\
\hline $8343.73(07)$ & 33.9700 & 1.3099 & 3.86 & 1.4026 & 33.3985 & 1.3099 & $0.572(02)$ \\
\hline $8344.20(07)$ & 37.5385 & 1.2379 & 3.30 & 1.3399 & 36.9657 & 1.2379 & $0.573(03)$ \\
\hline $8344.75(07)$ & 40.3905 & 1.2714 & 3.15 & 1.3807 & 39.8163 & 1.2714 & $0.574(04)$ \\
\hline $8345.23(07)$ & 46.1060 & 1.2319 & 2.67 & 1.3562 & 45.5306 & 1.2320 & $0.575(05)$ \\
\hline $8345.70(07)$ & 48.6691 & 1.1919 & 2.45 & 1.3228 & 48.0925 & 1.1919 & $0.577(07)$ \\
\hline $8346.12(07)$ & 53.8558 & 1.1712 & 2.17 & 1.3156 & 53.2782 & 1.1712 & $0.578(08)$ \\
\hline $8346.71(07)$ & 56.6905 & 1.1872 & 2.09 & 1.3390 & 56.1115 & 1.1873 & $0.579(09)$ \\
\hline $8347.25(07)$ & 62.3970 & 1.3015 & 2.09 & 1.4681 & 61.8169 & 1.3015 & $0.580(10)$ \\
\hline $8347.72(07)$ & 65.3705 & 1.1849 & 1.81 & 1.3592 & 64.7894 & 1.1849 & $0.581(11)$ \\
\hline $8348.26(07)$ & 73.6468 & 1.1839 & 1.61 & 1.3798 & 73.0646 & 1.1840 & $0.582(12)$ \\
\hline $8348.80(07)$ & 77.6169 & 1.2166 & 1.57 & 1.4228 & 77.0337 & 1.2167 & $0.583(13)$ \\
\hline $8349.39(07)$ & 83.2275 & 1.2775 & 1.53 & 1.4983 & 82.6433 & 1.2776 & $0.584(14)$ \\
\hline $8349.88(07)$ & 84.6464 & 1.1817 & 1.40 & 1.4062 & 84.0614 & 1.1818 & $0.585(15)$ \\
\hline $8350.36(07)$ & 89.4259 & 1.2367 & 1.38 & 1.4736 & 88.8401 & 1.2368 & $0.586(16)$ \\
\hline $8350.83(07)$ & 88.7259 & 1.2723 & 1.43 & 1.5074 & 88.1395 & 1.2724 & $0.586(16)$ \\
\hline $8351.31(07)$ & 92.1816 & 1.2068 & 1.31 & 1.4509 & 91.5946 & 1.2069 & $0.587(17)$ \\
\hline $8351.85(07)$ & 91.0746 & 1.3127 & 1.44 & 1.5539 & 90.4869 & 1.3129 & $0.588(18)$ \\
\hline $8352.32(07)$ & 91.7652 & 1.2537 & 1.37 & 1.4967 & 91.1771 & 1.2538 & $0.588(18)$ \\
\hline $8352.92(07)$ & 90.7730 & 1.2374 & 1.36 & 1.4778 & 90.1843 & 1.2375 & $0.589(19)$ \\
\hline $8353.40(07)$ & 88.6976 & 1.2258 & 1.38 & 1.4608 & 88.1086 & 1.2259 & $0.589(19)$ \\
\hline $8353.87(07)$ & 90.9187 & 1.2680 & 1.39 & 1.5088 & 90.3294 & 1.2682 & $0.589(19)$ \\
\hline $8354.35(07)$ & 88.4200 & 1.2682 & 1.43 & 1.5025 & 87.8305 & 1.2683 & $0.589(19)$ \\
\hline $8354.96(07)$ & 87.1118 & 1.2182 & 1.40 & 1.4491 & 86.5222 & 1.2184 & $0.590(20)$ \\
\hline $8355.43(07)$ & 86.1762 & 1.2732 & 1.48 & 1.5017 & 85.5866 & 1.2734 & $0.590(20)$ \\
\hline $8355.97(07)$ & 84.6883 & 1.2437 & 1.47 & 1.4683 & 84.0987 & 1.2438 & $0.590(20)$ \\
\hline $8356.45(07)$ & 85.1552 & 1.2510 & 1.47 & 1.4768 & 84.5658 & 1.2512 & $0.589(19)$ \\
\hline $8356.92(07)$ & 84.7208 & 1.5755 & 1.86 & 1.8001 & 84.1316 & 1.5756 & $0.589(19)$ \\
\hline $8357.40(07)$ & 81.5294 & 1.2262 & 1.50 & 1.4426 & 80.9405 & 1.2263 & $0.589(19)$ \\
\hline $8357.88(07)$ & 80.1758 & 1.2960 & 1.62 & 1.5089 & 79. & 1.2962 & $0.588(18)$ \\
\hline $8358.36(07)$ & 80.0005 & 1.2598 & 1.57 & 1.4722 & 79.4125 & 1.2599 & $0.588(18)$ \\
\hline $8358.83(07)$ & 77.9530 & 1.1855 & 1.52 & 1.3925 & 77.3657 & 1.1856 & $0.587(17)$ \\
\hline $8359.37(07)$ & 77.2138 & 1.2133 & 1.57 & 1.4184 & 76.6273 & 1.2134 & $0.587(17)$ \\
\hline $8359.79(07)$ & 76.1148 & 1.2178 & 1.60 & 1.4201 & 75.5289 & 1.2179 & $0.586(16)$ \\
\hline $8360.27(07)$ & 74.8982 & 1.2296 & 1.64 & 1.4287 & 74.3133 & 1.2297 & $0.585(15)$ \\
\hline $8360.82(07)$ & 74.0557 & 1.2609 & 1.70 & 1.4578 & 73.4719 & 1.2609 & $0.584(14)$ \\
\hline $8361.35(07)$ & 73.2283 & 1.2497 & 1.71 & 1.4445 & 72.6458 & 1.2498 & $0.583(12)$ \\
\hline $8361.77(07)$ & 72.6399 & 1.2015 & 1.65 & 1.3948 & 72.0585 & 1.2016 & $0.581(11)$ \\
\hline $8362.25(07)$ & 70.9479 & 1.1698 & 1.65 & 1.3587 & 70.3679 & 1.1699 & $0.580(10)$ \\
\hline $8362.79(07)$ & 69.6480 & 1.2151 & 1.74 & 1.4006 & 69.0697 & 1.2152 & $0.578(08)$ \\
\hline $8363.21(07)$ & 69.2431 & 1.2000 & 1.73 & 1.3844 & 68.6662 & 1.2000 & $0.577(07)$ \\
\hline $8363.68(07)$ & 69.4906 & 1.2508 & 1.80 & 1.4359 & 68.9156 & 1.2508 & $0.575(05)$ \\
\hline $8364.10(07)$ & 67.3170 & 1.2547 & 1.86 & 1.4341 & 66.7436 & 1.2547 & $0.573(03)$ \\
\hline $8364.58(07)$ & 65.9459 & 1.2072 & 1.83 & 1.3831 & 65.3745 & 1.2072 & $0.571(01)$ \\
\hline $8365.18(07)$ & 65.3861 & 1.2928 & 1.98 & 1.4672 & 64.8175 & 1.2928 & $0.569(01)$ \\
\hline $8365.60(07)$ & 65.6587 & 1.1522 & 1.75 & 1.3273 & 65.0921 & 1.1522 & $0.567(03)$ \\
\hline
\end{tabular}




\begin{tabular}{|c|c|c|c|c|c|c|c|}
\hline 8366.09(07) & 63.8118 & 1.3465 & 2.11 & 1.5168 & 63.2477 & 1.3465 & $0.564(06)$ \\
\hline 8366.56(07) & 61.0219 & 1.4998 & 2.46 & 1.6629 & 60.4604 & 1.4999 & $0.561(09)$ \\
\hline 8367.16(07) & 61.5844 & 1.2386 & 2.01 & 1.4031 & 61.0265 & 1.2387 & $0.558(12)$ \\
\hline $8367.64(07)$ & 61.1984 & 1.2298 & 2.01 & 1.3933 & 60.6435 & 1.2299 & $555(15)$ \\
\hline $8368.12(07)$ & 61.1377 & 1.1726 & 1.92 & 1.3360 & 60.5860 & 1.1728 & $0.552(18)$ \\
\hline 8368.60(07) & 57.5782 & 1.2211 & 2.12 & 1.3752 & 57.0298 & 1.2213 & $0.548(22)$ \\
\hline 8369.02(07) & 58.5712 & 1.2394 & 2.12 & 1.3961 & 58.0260 & 1.2397 & $0.545(25)$ \\
\hline 8369.50(07) & 57.4198 & 1.3116 & 2.28 & 1.4652 & 56.8782 & 1.3119 & $0.542(28)$ \\
\hline 8369.98(11) & 56.3743 & 1.9821 & 3.52 & 2.1330 & 55.8367 & 1.9823 & $0.538(32)$ \\
\hline 8370.03(08) & 56.8152 & 1.5918 & 2.80 & 1.7439 & 56.2780 & 1.5922 & $0.537(33)$ \\
\hline $8370.51(07)$ & 54.6853 & 1.2314 & 2.25 & 1.3780 & 54.1522 & 1.2320 & $0.533(37)$ \\
\hline $8371.00(07)$ & 55.5947 & 1.2621 & 2.27 & 1.4111 & 55.0659 & 1.2628 & $0.529(41)$ \\
\hline $8371.54(07)$ & 55.1509 & 1.3258 & 2.40 & 1.4735 & 54.6273 & 1.3266 & $0.524(46)$ \\
\hline $8372.02(07)$ & 52.9229 & 1.2654 & 2.39 & 1.4074 & 52.4041 & 1.2665 & $0.519(51)$ \\
\hline 8372.56(07) & 52.7617 & 1.2587 & 2.39 & 1.4003 & 52.2485 & 1.2600 & $.513(57)$ \\
\hline $8373.10(07)$ & 51.9624 & 1.2309 & 2.37 & 1.3704 & 51.4551 & 1.2325 & $0.507(63)$ \\
\hline 8373.64(07) & 52.3153 & 1.1675 & 2.23 & 1.3079 & 51.8142 & 1.1695 & $0.501(69)$ \\
\hline 8374.12(07) & 52.6383 & 1.2267 & 2.33 & 1.3679 & 52.1430 & 1.2290 & $0.495(75)$ \\
\hline 8374.66(07) & 51.9088 & 1.2087 & 2.33 & 1.3480 & 51.4174 & 1.2113 & $0.491(79)$ \\
\hline $8375.14(07)$ & 50.6279 & 1.2691 & 2.51 & 1.4051 & 50.1359 & 1.2715 & $0.492(78)$ \\
\hline $8375.62(07)$ & 50.2991 & 1.2485 & 2.48 & 1.3836 & 49.8065 & 1.2509 & $0.493(78)$ \\
\hline $8376.10(07)$ & 48.5488 & 1.3552 & 2.79 & 1.4858 & 48.0556 & 1.3574 & $0.493(77)$ \\
\hline $8376.71(07)$ & 48.7567 & 1.2117 & 2.49 & 1.3428 & 48.2628 & 1.2141 & $0.494(76)$ \\
\hline $8377.25(07)$ & 48.9887 & 1.3718 & 2.80 & 1.5035 & 48.4942 & 1.3739 & $0.495(75)$ \\
\hline 8377.73(07) & 48.4460 & 1.2173 & 2.51 & 1.3477 & 47.9509 & 1.2197 & $0.495(75)$ \\
\hline $8378.27(07)$ & 49.5580 & 1.1988 & 2.42 & 1.3320 & 49.0622 & 1.2011 & $0.496(74)$ \\
\hline 8378.93(07) & 49.6491 & 1.1988 & 2.41 & 1.3323 & 49.1525 & 1.2011 & $0.497(73)$ \\
\hline 8380.91(07) & 50.0272 & 1.1565 & 2.31 & 1.2909 & 49.5281 & 1.1587 & $0.499(71)$ \\
\hline 8382.96(07) & 52.0996 & 1.1851 & 2.27 & 1.3249 & 51.5980 & 1.1870 & $0.502(68)$ \\
\hline 8385.00(08) & 54.9023 & 1.4512 & 2.64 & 1.5983 & 54.3981 & 1.4527 & $0.504(66)$ \\
\hline $8385.06(11)$ & 54.1386 & 2.0900 & 3.86 & 2.2351 & 53.6343 & 2.0910 & $0.504(66)$ \\
\hline 8386.99(07) & 59.2067 & 1.1865 & 2.00 & 1.3448 & 58.7000 & 1.1882 & $0.507(63)$ \\
\hline 8388.98(07) & 59.3712 & 1.1451 & 1.93 & 1.3038 & 58.8621 & 1.1467 & $0.509(61)$ \\
\hline 8390.96(07) & 61.7334 & 1.1786 & 1.91 & 1.3435 & 61.2218 & 1.1801 & $0.512(58)$ \\
\hline 8392.90(07) & 59.9623 & 1.2133 & 2.02 & 1.3736 & 59.4483 & 1.2146 & $0.514(56)$ \\
\hline 8394.83(07) & 59.6027 & 1.1038 & 1.85 & 1.2631 & 59.0864 & 1.1051 & $0.516(54)$ \\
\hline 8396.75(07) & 62.0685 & 1.1308 & 1.82 & 1.2966 & 61.5498 & 1.1320 & $0.519(51)$ \\
\hline $8398.75(07)$ & 63.5010 & 1.1425 & 1.80 & 1.3119 & 62.9799 & 1.1435 & $0.521(49)$ \\
\hline $8400.74(07)$ & 60.9090 & 1.1577 & 1.90 & 1.3205 & 60.3854 & 1.1586 & $0.524(46)$ \\
\hline 8402.74(07) & 61.6223 & 1.1259 & 1.83 & 1.2905 & 61.0963 & 1.1268 & $0.526(44)$ \\
\hline 8404.80(07) & 60.7102 & 1.1304 & 1.86 & 1.2926 & 60.1817 & 1.1311 & $0.529(41)$ \\
\hline 8406.79(07) & 58.7294 & 1.2704 & 2.16 & 1.4275 & 58.1984 & 1.2710 & $0.531(39)$ \\
\hline 8408.98(07) & 59.1480 & 1.1370 & 1.92 & 1.2952 & 58.6143 & 1.1376 & $0.534(36)$ \\
\hline 8411.03(07) & 56.2751 & 1.4833 & 2.64 & 1.6339 & 55.7389 & 1.4836 & $0.536(34)$ \\
\hline 8412.97(07) & 54.6241 & 1.1249 & 2.06 & 1.2713 & 54.0855 & 1.1253 & $0.539(31)$ \\
\hline 8415.03(07) & 55.6656 & 1.1544 & 2.07 & 1.3035 & 55.1245 & 1.1547 & $0.541(29)$ \\
\hline
\end{tabular}




\begin{tabular}{|c|c|c|c|c|c|c|c|}
\hline 8417.03(07) & 55.8238 & 1.1069 & 1.98 & 1.2565 & 55.2804 & 1.1073 & $0.543(27)$ \\
\hline 8419.04(07) & 55.9318 & 1.2176 & 2.18 & 1.3674 & 55.3859 & 1.2178 & $0.546(24)$ \\
\hline 8420.98(07) & 54.5263 & 1.2023 & 2.21 & 1.3485 & 53.9781 & 1.2025 & $0.548(22)$ \\
\hline 8422.92(07) & 55.3984 & 1.2376 & 2.23 & 1.3860 & 54.8478 & 1.2377 & $0.551(19)$ \\
\hline $8424.75(07)$ & 52.7141 & 1.1811 & 2.24 & 1.3225 & 52.1613 & 1.1812 & $553(17)$ \\
\hline $8426.75(07)$ & 52.1986 & 1.1812 & 2.26 & 1.3212 & 51.6435 & 1.1813 & $0.555(15)$ \\
\hline 8428.76(07) & 52.1546 & 1.1472 & 2.20 & 1.2871 & 51.5970 & 1.1472 & $0.558(12)$ \\
\hline $8430.65(08)$ & 50.6725 & 1.5808 & 3.12 & 1.7169 & 50.1126 & 1.5808 & $0.560(10)$ \\
\hline $8430.71(11)$ & 52.8623 & 2.1120 & 4.00 & 2.2538 & 52.3023 & 2.1120 & $0.560(10)$ \\
\hline 8432.66(07) & 50.3002 & 1.2577 & 2.50 & 1.3928 & 49.7379 & 1.2577 & $0.562(08)$ \\
\hline 8434.80(07) & 50.4740 & 1.3249 & 2.62 & 1.4605 & 49.9092 & 1.3249 & $0.565(05)$ \\
\hline $8436.74(07)$ & 50.1038 & 1.1770 & 2.35 & 1.3116 & 49.5367 & 1.1770 & $0.567(03)$ \\
\hline 8438.81(07) & 50.5854 & 1.2194 & 2.41 & 1.3553 & 50.0158 & 1.2194 & $0.570(00)$ \\
\hline 8440.95(07) & 52.1534 & 1.1757 & 2.25 & 1.3157 & 51.5813 & 1.1757 & $0.572(02)$ \\
\hline 8442.90(07) & 49.6080 & 1.1898 & 2.40 & 1.3231 & 49.0335 & 1.1898 & $.574(04)$ \\
\hline 8444.99(07) & 49.3824 & 1.0897 & 2.21 & 1.2224 & 48.8055 & 1.0897 & $0.577(07)$ \\
\hline 8447.06(07) & 51.0791 & 1.1351 & 2.22 & 1.2723 & 50.4997 & 1.1352 & $0.579(09)$ \\
\hline 8448.89(07) & 50.3459 & 1.2240 & 2.43 & 1.3592 & 49.7657 & 1.2240 & $0.580(10)$ \\
\hline $8450.92(07)$ & 51.2372 & 1.1220 & 2.19 & 1.2596 & 50.6572 & 1.1220 & $0.580(10)$ \\
\hline 8452.93(07) & 53.0569 & 1.1567 & 2.18 & 1.2990 & 52.4772 & 1.1567 & $0.580(10)$ \\
\hline 8454.76(07) & 52.1036 & 1.1136 & 2.14 & 1.2535 & 51.5241 & 1.1137 & $0.579(09)$ \\
\hline 8456.67(07) & 52.1788 & 1.1048 & 2.12 & 1.2449 & 51.5995 & 1.1049 & $0.579(09)$ \\
\hline $8458.75(07)$ & 51.8489 & 1.1347 & 2.19 & 1.2739 & 51.2699 & 1.1348 & $0.579(09)$ \\
\hline $8460.65(07)$ & 51.3717 & 1.1658 & 2.27 & 1.3037 & 50.7930 & 1.1658 & $0.579(09)$ \\
\hline 8462.68(07) & 53.8418 & 1.1257 & 2.09 & 1.2701 & 53.2633 & 1.1258 & $0.578(08)$ \\
\hline 8464.82(07) & 52.6958 & 1.2733 & 2.42 & 1.4146 & 52.1176 & 1.2733 & $0.578(08)$ \\
\hline 8466.79(07) & 51.9342 & 1.1461 & 2.21 & 1.2855 & 51.3562 & 1.1462 & $0.578(08)$ \\
\hline 8468.88(07) & 52.6604 & 1.1812 & 2.24 & 1.3225 & 52.0828 & 1.1812 & $0.578(08)$ \\
\hline 8470.96(07) & 51.8862 & 1.1302 & 2.18 & 1.2695 & 51.3088 & 1.1302 & $0.577(07)$ \\
\hline 8472.94(07) & 52.1731 & 1.1276 & 2.16 & 1.2676 & 51.5960 & 1.1276 & $0.577(07)$ \\
\hline $8475.02(07)$ & 53.7014 & 1.1416 & 2.13 & 1.2856 & 53.1245 & 1.1416 & $0.577(07)$ \\
\hline 8477.06(07) & 52.9416 & 1.1852 & 2.24 & 1.3272 & 52.3650 & 1.1852 & $0.577(07)$ \\
\hline 8479.03(07) & 53.5673 & 1.0639 & 1.99 & 1.2075 & 52.9910 & 1.0639 & $0.576(06)$ \\
\hline 8481.00(07) & 54.1463 & 1.1300 & 2.09 & 1.2751 & 53.5702 & 1.1300 & $0.576(06)$ \\
\hline 8483.04(07) & 55.0475 & 1.1343 & 2.06 & 1.2818 & 54.4717 & 1.1343 & $0.576(06)$ \\
\hline $8484.88(07)$ & 53.1032 & 1.1573 & 2.18 & 1.2998 & 52.5276 & 1.1573 & $0.576(06)$ \\
\hline 8486.79(07) & 54.3096 & 1.0653 & 1.96 & 1.2109 & 53.7342 & 1.0653 & $0.575(05)$ \\
\hline 8488.84(07) & 53.6157 & 1.1532 & 2.15 & 1.2970 & 53.0406 & 1.1532 & $0.575(05)$ \\
\hline 8490.81(07) & 53.2181 & 1.1131 & 2.09 & 1.2559 & 52.6432 & 1.1131 & $0.575(05)$ \\
\hline 8492.73(07) & 52.7469 & 1.1834 & 2.24 & 1.3249 & 52.1723 & 1.1834 & $0.575(05)$ \\
\hline 8494.77(07) & 54.6618 & 1.1297 & 2.07 & 1.2762 & 54.0874 & 1.1297 & $0.574(04)$ \\
\hline 8496.81(07) & 53.4991 & 1.1404 & 2.13 & 1.2838 & 52.9250 & 1.1404 & $0.574(04)$ \\
\hline 8498.85(07) & 54.5962 & 1.1192 & 2.05 & 1.2655 & 54.0224 & 1.1192 & $0.574(04)$ \\
\hline $8500.95(07)$ & 55.3497 & 1.1205 & 2.02 & 1.2688 & 54.7760 & 1.1205 & $0.574(04)$ \\
\hline 8503.07(07) & 54.9742 & 1.1260 & 2.05 & 1.2733 & 54.4006 & 1.1260 & $0.574(04)$ \\
\hline $8505.05(07)$ & 53.7822 & 1.1181 & 2.08 & 1.2623 & 53.2086 & 1.1181 & $0.574(04)$ \\
\hline
\end{tabular}




\begin{tabular}{|c|c|c|c|c|c|c|c|}
\hline $8507.15(07)$ & 55.7045 & 1.3394 & 2.40 & 1.4886 & 55.1311 & 1.3394 & $0.573(04)$ \\
\hline 8509.14(07) & 54.4228 & 1.0569 & 1.94 & 1.2028 & 53.8494 & 1.0569 & $0.573(04)$ \\
\hline 8511.06(07) & 53.8470 & 1.1001 & 2.04 & 1.2444 & 53.2736 & 1.1001 & $0.573(04)$ \\
\hline 8513.05(07) & 52.3176 & 1.1236 & 2.15 & 1.2640 & 51.7443 & 1.1236 & $0.573(04)$ \\
\hline $8515.04(07)$ & 53.6173 & 1.0595 & 1.98 & 1.2033 & 53.0442 & 1.0595 & $0.573(04)$ \\
\hline $8516.84(07)$ & 53.9073 & 1.1218 & 2.08 & 1.2663 & 53.3343 & 1.1218 & $0.573(04)$ \\
\hline $8519.08(05)$ & 53.7852 & 0.8248 & 1.53 & 0.9690 & 53.2122 & 0.8248 & $0.573(04)$ \\
\hline $8529.10(07)$ & 48.2038 & 1.2008 & 2.49 & 1.3305 & 47.6312 & 1.2008 & $0.573(04)$ \\
\hline 8539.59(07) & 54.8657 & 1.0352 & 1.89 & 1.1822 & 54.2935 & 1.0352 & $0.572(04)$ \\
\hline $8549.29(07)$ & 42.3568 & 1.0655 & 2.52 & 1.1800 & 41.7850 & 1.0655 & $0.572(05)$ \\
\hline $8559.27(07)$ & 46.6855 & 1.0645 & 2.28 & 1.1902 & 46.1141 & 1.0645 & $0.571(05)$ \\
\hline $8569.58(07)$ & 44.7834 & 1.1368 & 2.54 & 1.2576 & 44.2124 & 1.1368 & $0.571(05)$ \\
\hline $8579.29(07)$ & 48.3248 & 1.1274 & 2.33 & 1.2574 & 47.7542 & 1.1274 & $0.571(05)$ \\
\hline 8589.34(07) & 48.6735 & 1.0830 & 2.23 & 1.2140 & 48.1034 & 1.0831 & $0.570(05)$ \\
\hline 8599.66(05) & 53.8066 & 0.8024 & 1.49 & 0.9466 & 53.2369 & 0.8024 & $0.570(05)$ \\
\hline 8609.57(07) & 42.2109 & 1.4014 & 3.32 & 1.5155 & 41.6416 & 1.4014 & $0.569(06)$ \\
\hline $8619.50(07)$ & 46.9062 & 1.1391 & 2.43 & 1.2654 & 46.3373 & 1.1391 & $0.569(06)$ \\
\hline 8639.86(07) & 39.6496 & 1.0536 & 2.66 & 1.1611 & 39.0816 & 1.0536 & $0.568(06)$ \\
\hline 8659.76(07) & 45.2057 & 1.0719 & 2.37 & 1.1938 & 44.6385 & 1.0719 & $0.567(06)$ \\
\hline 8679.56(11) & 44.5396 & 1.9369 & 4.35 & 2.0571 & 43.9733 & 1.9369 & $0.566(07)$ \\
\hline 8679.63(08) & 42.3320 & 1.3253 & 3.13 & 1.4397 & 41.7657 & 1.3253 & $0.566(07)$ \\
\hline 8699.91(07) & 49.0839 & 1.0717 & 2.18 & 1.2037 & 48.5184 & 1.0718 & $0.565(07)$ \\
\hline 8719.62(07) & 44.2130 & 1.0692 & 2.42 & 1.1885 & 43.6485 & 1.0692 & $0.565(07)$ \\
\hline 8739.71(07) & 49.8887 & 1.1325 & 2.27 & 1.2666 & 49.3250 & 1.1326 & $0.564(08)$ \\
\hline 8759.82(07) & 47.9584 & 1.1484 & 2.39 & 1.2774 & 47.3957 & 1.1484 & $0.563(08)$ \\
\hline $8779.56(05)$ & 52.5678 & 0.8076 & 1.54 & 0.9486 & 52.0060 & 0.8076 & $0.562(08)$ \\
\hline 8799.99(07) & 46.6305 & 1.1166 & 2.39 & 1.2422 & 46.0697 & 1.1166 & $0.561(09)$ \\
\hline 8819.84(07) & 51.5817 & 1.0752 & 2.08 & 1.2137 & 51.0218 & 1.0753 & $0.560(09)$ \\
\hline $8840.06(07)$ & 44.4631 & 1.6648 & 3.74 & 1.7847 & 43.9041 & 1.6648 & $0.559(09)$ \\
\hline 8870.37(07) & 52.7547 & 1.0818 & 2.05 & 1.2233 & 52.1972 & 1.0818 & $0.558(09)$ \\
\hline 8900.35(08) & 56.5121 & 1.4510 & 2.57 & 1.6023 & 55.9560 & 1.4510 & $0.556(10)$ \\
\hline 8930.46(05) & 56.2608 & 2.4498 & 4.35 & 2.6005 & 55.7062 & 2.4498 & $0.555(10)$ \\
\hline $8960.45(07)$ & 56.7287 & 1.2394 & 2.18 & 1.3913 & 56.1756 & 1.2395 & $0.553(11)$ \\
\hline $8990.29(12)$ & 55.1360 & 1.9125 & 3.47 & 2.0602 & 54.5844 & 1.9125 & $0.552(11)$ \\
\hline 8990.36(08) & 49.9196 & 1.4712 & 2.95 & 1.6053 & 49.3680 & 1.4712 & $0.552(11)$ \\
\hline $9020.47(07)$ & 63.1531 & 1.2094 & 1.91 & 1.3779 & 62.6029 & 1.2094 & $0.550(11)$ \\
\hline
\end{tabular}


Table S3. Measured X-ray mass attenuation coefficients $\left[{ }_{\rho}^{\mu}\right]_{S}$ of the bis(N-npropyl-salicylaldiminato) nickel(II) complex determined from the intensity measurements $\left(I_{0}\right.$ and $\left.I\right)$ with a $15 \mathrm{mM}$ solution of the complex, and its corresponding solvent. A number of experimental systematics including energy calibration, dark-current, solvent attenuations, harmonic contamination and thickness-ratio (from the actual solvent attenuation and fitted background of the sample (solution) were corrected to obtain the final result (weighted mean of multiple measurements at each energy). The first column lists calibrated energies (Section 4.3) in $\mathrm{eV}$, and their associated uncertainties in parentheses. The second, third and fourth columns present corrected total X-ray mass attenuation coefficients $\left[\frac{\mu}{\rho}\right]_{S}$ with associated relative and percentage uncertainties. The fifth column lists the absolute uncertainties including the uncertainty contributions from the thickness ratio $t_{\text {ratio }}$ and column density $[\rho t]_{c}$ measurements. The sixth and the seventh columns list the (effective) photoelectric absorption coefficient $\left[\frac{\mu}{\rho}\right]_{p e}$ determined by subtracting the tabulated X-ray mass attenuations of the complex for the Rayleigh and Compton scattering from the total experimental X-ray mass attenuation coefficients $\left[\frac{\mu}{\rho}\right]_{S}$ at measured energies, with their corresponding uncertainties. The eighth column lists the X-ray mass attenuations of Rayleigh and Compton scattering, where the corresponding uncertainty of $\left[\frac{\mu}{\rho}\right]_{R+C}$ was determined from half of the variation between FFAST and XCOM tabulated values.

\begin{tabular}{|c|c|c|c|c|c|c|c|}
\hline $\begin{array}{c}\text { Energy } \\
(\mathrm{eV})\end{array}$ & $\begin{array}{c}{\left[\frac{\mu}{\rho}\right]_{s}} \\
\left(\mathrm{~cm}^{2} / g\right)\end{array}$ & $\begin{array}{c}\sigma_{\left[\frac{\mu}{\rho}\right]_{r e l}} \\
\left(\mathrm{~cm}^{2} / g\right)\end{array}$ & $\begin{array}{c}\sigma_{\left[\frac{\mu}{\rho}\right]}(r e l) \\
(\%)\end{array}$ & $\begin{array}{c}\sigma_{\left[\frac{\mu}{\rho}\right]_{a b s}} \\
\left(\mathrm{~cm}^{2} / g\right)\end{array}$ & $\begin{array}{c}{\left[\frac{\mu}{\rho}\right]_{p e}} \\
\left(\mathrm{~cm}^{2} / g\right)\end{array}$ & $\begin{array}{c}\sigma_{\left[\frac{\mu}{\rho}\right]_{p e}} \\
\left(\mathrm{~cm}^{2} / \mathrm{g}\right)\end{array}$ & $\begin{array}{l}{\left[\frac{\mu}{\rho}\right]_{R+C}} \\
\left(\mathrm{~cm}^{2} / \mathrm{g}\right)\end{array}$ \\
\hline $7918.60(11)$ & 15.0026 & 0.3439 & 2.29 & 0.3566 & 14.3975 & 0.3440 & $0.605(10)$ \\
\hline 7958.94(10) & 14.4521 & 0.1280 & 0.89 & 0.1404 & 13.8570 & 0.1280 & $0.595(02)$ \\
\hline $7998.73(10)$ & 12.9877 & 0.2331 & 1.79 & 0.2446 & 12.4021 & 0.2331 & $0.586(05)$ \\
\hline $8039.15(10)$ & 13.4378 & 0.1575 & 1.17 & 0.1693 & 12.8614 & 0.1579 & $0.576(12)$ \\
\hline $8078.88(12)$ & 12.1499 & 0.2654 & 2.18 & 0.2765 & 11.5823 & 0.2661 & $0.568(19)$ \\
\hline $8078.93(17)$ & 11.3425 & 0.2010 & 1.77 & 0.2117 & 10.7749 & 0.2019 & $0.568(19)$ \\
\hline $8119.13(10)$ & 11.8420 & 0.1011 & 0.85 & 0.1120 & 11.2833 & 0.1043 & $0.559(26)$ \\
\hline $8159.17(09)$ & 11.3740 & 0.1753 & 1.54 & 0.1859 & 10.8239 & 0.1782 & $0.550(32)$ \\
\hline 8199.09(09) & 11.9725 & 0.1063 & 0.89 & 0.1173 & 11.4329 & 0.1137 & $0.540(40)$ \\
\hline $8239.54(09)$ & 9.8658 & 0.2582 & 2.62 & 2680 & 9.3359 & 0.2631 & $0.530(50)$ \\
\hline $8278.65(09)$ & 11.1380 & 0.0897 & 0.81 & 0.1002 & 10.6173 & 0.1075 & $0.521(59)$ \\
\hline $8283.63(09)$ & 10.9600 & 0.1474 & 1.34 & 0.1578 & 10.4404 & 0.1593 & $0.520(60)$ \\
\hline $8288.75(09)$ & 11.4219 & 0.0855 & 0.75 & 0.0962 & 10.9036 & 0.1055 & $0.518(62)$ \\
\hline $8293.87(09)$ & 10.9533 & 0.1427 & 1.30 & 0.1532 & 10.4362 & 0.1560 & $0.517(63)$ \\
\hline 8298.76(09) & 11.3262 & 0.0912 & 0.81 & 0.1018 & 10.8103 & 0.1115 & $0.516(64)$ \\
\hline $8303.65(05)$ & 11.9663 & 0.0625 & 0.52 & 0.0735 & 11.4517 & 0.0905 & $0.515(65)$ \\
\hline $8308.43(09)$ & 12.0490 & 0.0994 & 0.82 & 0.1104 & 11.5356 & 0.1197 & $0.513(67)$ \\
\hline $8309.38(09)$ & 12.2179 & 0.0853 & 0.70 & 0.0965 & 11.7047 & 0.1084 & $0.513(67)$ \\
\hline $8310.32(09)$ & 11.9839 & 0.0917 & 0.77 & 0.1027 & 11.4710 & 0.1136 & $0.513(67)$ \\
\hline 8311.34(09) & 12.2849 & 0.0927 & 0.75 & 0.1039 & 11.7723 & 0.1146 & $0.513(67)$ \\
\hline 8312.34(09) & 12.2484 & 0.1002 & 0.82 & 0.1113 & 11.7360 & 0.1208 & $0.512(68)$ \\
\hline $8313.46(09)$ & 12.6040 & 0.0935 & 0.74 & 0.1049 & 12.0918 & 0.1156 & $0.512(68)$ \\
\hline $8314.46(09)$ & 12.5348 & 0.0891 & 0.7 & 0.1004 & 12.0229 & 0.1122 & $0.512(68)$ \\
\hline $8315.41(09)$ & 12.5665 & 0.0851 & 0.68 & 0.0965 & 12.0549 & 0.1092 & $0.512(68)$ \\
\hline
\end{tabular}




\begin{tabular}{|c|c|c|c|c|c|c|c|}
\hline 8316.42(09) & 12.6117 & 0.0943 & 0.75 & 0.1057 & 12.1003 & 0.1166 & $0.511(69)$ \\
\hline 8317.49(09) & 12.7160 & 0.0884 & 0.70 & 0.0998 & 12.2049 & 0.1121 & $0.511(69)$ \\
\hline $8318.49(09)$ & 12.8593 & 0.0867 & 0.67 & 0.0982 & 12.3484 & 0.1109 & $0.511(69)$ \\
\hline 8319.02(09) & 13.2490 & 0.0919 & 0.69 & 0.1036 & 12.7383 & 0.1151 & $0.511(69)$ \\
\hline 8319.62(09) & 13.3094 & 0.0805 & 0.60 & 0.0922 & 12.7988 & 0.1063 & $0.511(69)$ \\
\hline 8320.09(09) & 13.1921 & 0.0964 & 0.73 & 0.1081 & 12.6816 & 0.1189 & $0.510(70)$ \\
\hline 8320.62(09) & 13.0655 & 0.1029 & 0.79 & 0.1145 & 12.5552 & 0.1243 & $0.510(70)$ \\
\hline 8321.10(09) & 12.9936 & 0.1003 & 0.77 & 0.1119 & 12.4834 & 0.1222 & $0.510(70)$ \\
\hline 8321.58(09) & 13.2144 & 0.1411 & 1.07 & 0.1528 & 12.7043 & 0.1575 & $0.510(70)$ \\
\hline 8322.05(09) & 13.5160 & 0.0928 & 0.69 & 0.1047 & 13.0060 & 0.1163 & $0.510(70)$ \\
\hline 8322.58(09) & 13.2740 & 0.1103 & 0.83 & 0.1220 & 12.7641 & 0.1307 & $0.510(70)$ \\
\hline 8323.12(09) & 13.2916 & 0.0993 & 0.75 & 0.1110 & 12.7819 & 0.1217 & $0.510(70)$ \\
\hline 8323.59(09) & 13.1612 & 0.0988 & 0.75 & 0.1104 & 12.6516 & 0.1213 & $0.510(70)$ \\
\hline 8324.12(09) & 13.1028 & 0.0902 & 0.69 & 0.1019 & 12.5933 & 0.1145 & $0.509(71)$ \\
\hline $8324.65(09)$ & 13.0729 & 0.0946 & 0.72 & 0.1062 & 12.5628 & 0.1177 & $0.510(70)$ \\
\hline 8325.13(09) & 12.7662 & 0.0912 & 0.71 & 0.1026 & 12.2543 & 0.1138 & $0.512(68)$ \\
\hline 8325.66(09) & 12.6681 & 0.1017 & 0.80 & 0.1131 & 12.1542 & 0.1214 & $0.514(66)$ \\
\hline 8326.15(09) & 12.9941 & 0.0868 & 0.67 & 0.0983 & 12.4784 & 0.1080 & $0.516(64)$ \\
\hline 8326.62(09) & 13.3032 & 0.0842 & 0.63 & 0.0960 & 12.7858 & 0.1049 & $0.517(63)$ \\
\hline $8327.15(09)$ & 12.9793 & 0.0863 & 0.66 & 0.0979 & 12.4599 & 0.1054 & $0.519(61)$ \\
\hline 8327.57(09) & 12.7948 & 0.0924 & 0.72 & 0.1039 & 12.2737 & 0.1096 & $0.521(59)$ \\
\hline 8328.04(09) & 12.7191 & 0.0907 & 0.71 & 0.1021 & 12.1963 & 0.1072 & $0.523(57)$ \\
\hline 8328.57(09) & 12.5894 & 0.0968 & 0.77 & 0.1082 & 12.0645 & 0.1114 & $0.525(55)$ \\
\hline 8329.05(09) & 12.9246 & 0.0853 & 0.66 & 0.0968 & 12.3979 & 0.1006 & $0.527(53)$ \\
\hline 8329.52(09) & 12.7369 & 0.0904 & 0.71 & 0.1018 & 12.2085 & 0.1041 & $0.528(52)$ \\
\hline 8330.00(09) & 12.7613 & 0.1239 & 0.97 & 0.1353 & 12.2310 & 0.1335 & $0.530(50)$ \\
\hline 8330.47(09) & 12.9064 & 0.1028 & 0.80 & 0.1143 & 12.3744 & 0.1135 & $0.532(48)$ \\
\hline 8331.07(09) & 12.9967 & 0.0939 & 0.72 & 0.1055 & 12.4626 & 0.1045 & $0.534(46)$ \\
\hline 8331.55(09) & 13.1421 & 0.0943 & 0.72 & 0.1060 & 12.6062 & 0.1041 & $0.536(44)$ \\
\hline 8331.96(09) & 12.9859 & 0.0871 & 0.67 & 0.0987 & 12.4484 & 0.0969 & $0.537(43)$ \\
\hline 8332.50(09) & 13.2308 & 0.0871 & 0.66 & 0.0988 & 12.6913 & 0.0960 & $0.539(41)$ \\
\hline 8332.91(09) & 13.2972 & 0.0848 & 0.64 & 0.0965 & 12.7562 & 0.0933 & $0.541(39)$ \\
\hline 8333.45(09) & 13.4960 & 0.0815 & 0.60 & 0.0933 & 12.9581 & 0.0875 & $0.538(32)$ \\
\hline 8333.86(09) & 13.7092 & 0.0983 & 0.72 & 0.1102 & 13.1698 & 0.1029 & $0.539(31)$ \\
\hline 8334.34(09) & 13.8201 & 0.0883 & 0.64 & 0.1003 & 13.2789 & 0.0929 & $0.541(29)$ \\
\hline 8334.87(09) & 13.8094 & 0.1021 & 0.74 & 0.1141 & 13.2664 & 0.1056 & $0.543(27)$ \\
\hline 8335.29(09) & 13.9741 & 0.0879 & 0.63 & 0.1000 & 13.4296 & 0.0915 & $0.545(25)$ \\
\hline 8335.82(09) & 14.1363 & 0.0855 & 0.60 & 0.0977 & 13.5899 & 0.0887 & $0.546(24)$ \\
\hline 8336.31(09) & 14.0868 & 0.0850 & 0.60 & 0.0972 & 13.5387 & 0.0878 & $0.548(22)$ \\
\hline 8336.84(09) & 14.2042 & 0.1017 & 0.72 & 0.1140 & 13.6542 & 0.1037 & $0.550(20)$ \\
\hline 8337.38(09) & 14.4006 & 0.0889 & 0.62 & 0.1012 & 13.8488 & 0.0907 & $0.552(18)$ \\
\hline $8337.85(09)$ & 14.8022 & 0.0926 & 0.63 & 0.1052 & 14.2488 & 0.0941 & $0.553(17)$ \\
\hline 8338.33(09) & 15.4063 & 0.0885 & 0.57 & 0.1015 & 14.8512 & 0.0898 & $0.555(15)$ \\
\hline 8338.80(09) & 15.8844 & 0.0924 & 0.58 & 0.1057 & 15.3278 & 0.0934 & $0.557(13)$ \\
\hline 8339.28(09) & 16.5746 & 0.0839 & 0.51 & 0.0975 & 16.0164 & 0.0847 & $0.558(12)$ \\
\hline $8339.75(09)$ & 17.1886 & 0.0850 & 0.49 & 0.0990 & 16.6289 & 0.0857 & $0.560(10)$ \\
\hline
\end{tabular}




\begin{tabular}{|c|c|c|c|c|c|c|c|}
\hline $8340.23(09)$ & 17.9072 & 0.0882 & 0.49 & 0.1025 & 17.3460 & 0.0886 & $0.561(09)$ \\
\hline 8340.76(09) & 18.8303 & 0.0964 & 0.51 & 0.1113 & 18.2675 & 0.0966 & $0.563(07)$ \\
\hline 8341.30(09) & 20.0802 & 0.0859 & 0.43 & 0.1015 & 19.5157 & 0.0861 & $0.565(06)$ \\
\hline 8341.72(09) & 20.9746 & 0.0879 & 0.42 & 0.1041 & 20.4088 & 0.0880 & $0.566(04)$ \\
\hline 8342.26(09) & 22.3591 & 0.0869 & 0.39 & 0.1038 & 21.7918 & 0.0870 & $0.567(03)$ \\
\hline $8342.85(09)$ & 24.1739 & 0.0978 & 0.40 & 0.1158 & 23.6048 & 0.0978 & $0.569(01)$ \\
\hline 8343.39(09) & 26.6625 & 0.0865 & 0.32 & 0.1059 & 26.0919 & 0.0865 & $0.571(01)$ \\
\hline 8343.86(09) & 28.6822 & 0.0880 & 0.31 & 0.1085 & 28.1103 & 0.0880 & $0.572(02)$ \\
\hline 8344.40(09) & 31.8424 & 0.0945 & 0.30 & 0.1168 & 31.2691 & 0.0946 & $0.573(03)$ \\
\hline 8344.88(09) & 34.6921 & 0.0834 & 0.24 & 0.1074 & 34.1175 & 0.0836 & $0.575(05)$ \\
\hline 8345.35(09) & 38.6115 & 0.0930 & 0.24 & 0.1192 & 38.0358 & 0.0932 & $0.576(06)$ \\
\hline 8345.83(09) & 42.3968 & 0.0798 & 0.19 & 0.1081 & 41.8199 & 0.0801 & $0.577(07)$ \\
\hline $8346.42(09)$ & 47.0804 & 0.0929 & 0.20 & 0.1239 & 46.5021 & 0.0933 & $0.578(08)$ \\
\hline 8346.91(09) & 51.3041 & 0.0932 & 0.18 & 0.1266 & 50.7247 & 0.0936 & $0.579(09)$ \\
\hline 8347.44(09) & 56.3805 & 0.0962 & 0.17 & 0.1325 & 55.7999 & 0.0968 & $0.581(11)$ \\
\hline 8348.52(09) & 70.4395 & 0.0918 & 0.13 & 0.1361 & 69.8568 & 0.0927 & $0.583(13)$ \\
\hline 8350.60(09) & 76.0340 & 0.0945 & 0.12 & 0.1420 & 75.4479 & 0.0958 & $0.586(16)$ \\
\hline 8352.64(09) & 75.0248 & 0.0917 & 0.12 & 0.1386 & 74.4364 & 0.0935 & $0.588(18)$ \\
\hline 8354.67(09) & 72.4205 & 0.0935 & 0.13 & 0.1389 & 71.8309 & 0.0955 & $0.590(20)$ \\
\hline $8356.70(09)$ & 68.0119 & 0.0848 & 0.12 & 0.1278 & 67.4226 & 0.0870 & $0.589(19)$ \\
\hline 8358.61(09) & 64.6053 & 0.1025 & 0.16 & 0.1434 & 64.0177 & 0.1040 & $0.588(18)$ \\
\hline $8360.59(08)$ & 61.3634 & 0.0921 & 0.15 & 0.1313 & 60.7791 & 0.0932 & $0.584(14)$ \\
\hline 8362.57(08) & 58.7797 & 0.0828 & 0.14 & 0.1204 & 58.2007 & 0.0833 & $0.579(09)$ \\
\hline 8364.36(08) & 55.9661 & 0.0913 & 0.16 & 0.1274 & 55.3938 & 0.0914 & $0.572(02)$ \\
\hline 8366.39(08) & 54.2395 & 0.0904 & 0.17 & 0.1254 & 53.6771 & 0.0907 & $0.562(08)$ \\
\hline 8368.38(08) & 52.1884 & 0.0836 & 0.16 & 0.1175 & 51.6385 & 0.0860 & $0.550(20)$ \\
\hline $8370.29(08)$ & 50.6032 & 0.0935 & 0.18 & 0.1265 & 50.0682 & 0.0998 & $0.535(35)$ \\
\hline $8372.34(08)$ & 48.6696 & 0.0872 & 0.18 & 0.1191 & 48.1540 & 0.1028 & $0.516(54)$ \\
\hline 8374.38(08) & 47.8563 & 0.1010 & 0.21 & 0.1325 & 47.3642 & 0.1276 & $0.492(78)$ \\
\hline $8376.42(08)$ & 47.2438 & 0.0976 & 0.21 & 0.1287 & 46.7503 & 0.1240 & $0.494(77)$ \\
\hline 8378.53(08) & 47.8057 & 0.1334 & 0.28 & 0.1648 & 47.3096 & 0.1525 & $0.496(74)$ \\
\hline $8380.57(08)$ & 49.1540 & 0.0861 & 0.18 & 0.1183 & 48.6553 & 0.1118 & $0.499(71)$ \\
\hline $8382.62(08)$ & 51.1068 & 0.0931 & 0.18 & 0.1264 & 50.6055 & 0.1157 & $0.501(69)$ \\
\hline $8384.66(08)$ & 52.9162 & 0.0932 & 0.18 & 0.1275 & 52.4124 & 0.1143 & $0.504(66)$ \\
\hline 8386.64(08) & 54.2970 & 0.1060 & 0.20 & 0.1411 & 53.7908 & 0.1237 & $0.506(64)$ \\
\hline 8388.58(08) & 56.0695 & 0.0917 & 0.16 & 0.1278 & 55.5608 & 0.1103 & $0.509(61)$ \\
\hline $8390.56(08)$ & 56.9979 & 0.0924 & 0.16 & 0.1290 & 56.4868 & 0.1096 & $0.511(59)$ \\
\hline 8392.56(08) & 58.0661 & 0.0770 & 0.13 & 0.1142 & 57.5525 & 0.0955 & $0.514(56)$ \\
\hline 8394.43(08) & 58.5039 & 0.1424 & 0.24 & 0.1799 & 57.9881 & 0.1524 & $0.516(54)$ \\
\hline 8396.35(08) & 59.0096 & 0.1540 & 0.26 & 0.1918 & 58.4913 & 0.1624 & $0.518(52)$ \\
\hline 8398.35(08) & 59.7412 & 0.0887 & 0.15 & 0.1269 & 59.2205 & 0.1015 & $0.521(49)$ \\
\hline $8400.34(08)$ & 59.7195 & 0.1038 & 0.17 & 0.1420 & 59.1963 & 0.1139 & $0.523(47)$ \\
\hline 8402.33(08) & 60.3301 & 0.0926 & 0.15 & 0.1311 & 59.8045 & 0.1027 & $0.526(44)$ \\
\hline $8404.40(08)$ & 59.6788 & 0.1113 & 0.19 & 0.1494 & 59.1507 & 0.1189 & $0.528(42)$ \\
\hline $8406.45(08)$ & 59.6471 & 0.0840 & 0.14 & 0.1222 & 59.1165 & 0.0928 & $0.531(39)$ \\
\hline $8408.51(08)$ & 58.4479 & 0.1612 & 0.28 & 0.1987 & 57.9148 & 0.1654 & $0.533(37)$ \\
\hline
\end{tabular}




\begin{tabular}{|c|c|c|c|c|c|c|c|}
\hline 8410.63(08) & 58.2696 & 0.1199 & 0.21 & 0.1573 & 57.7339 & 0.1247 & $0.536(34)$ \\
\hline $8412.64(08)$ & 57.2181 & 0.1248 & 0.22 & 0.1616 & 56.6799 & 0.1288 & $0.538(32)$ \\
\hline $8414.69(08)$ & 56.5993 & 0.1021 & 0.18 & 0.1385 & 56.0587 & 0.1063 & $0.541(29)$ \\
\hline $8416.69(08)$ & 56.1816 & 0.1064 & 0.19 & 0.1426 & 55.6385 & 0.1098 & $0.543(27)$ \\
\hline $8418.58(08)$ & 56.0481 & 0.1152 & 0.21 & 0.1513 & 55.5027 & 0.1178 & $0.545(25)$ \\
\hline $8420.58(08)$ & 55.9638 & 0.0862 & 0.15 & 0.1223 & 55.4160 & 0.0891 & $0.548(22)$ \\
\hline $8422.53(08)$ & 55.6392 & 0.1167 & 0.21 & 0.1525 & 55.0891 & 0.1183 & $0.550(20)$ \\
\hline $8424.35(08)$ & 55.2355 & 0.0901 & 0.16 & 0.1257 & 54.6832 & 0.0918 & $0.552(18)$ \\
\hline $8426.35(08)$ & 55.3025 & 0.1011 & 0.18 & 0.1368 & 54.7478 & 0.1023 & $0.555(15)$ \\
\hline $8428.37(08)$ & 55.1096 & 0.0855 & 0.16 & 0.1210 & 54.5525 & 0.0864 & $0.557(13)$ \\
\hline $8430.25(08)$ & 55.0282 & 0.0775 & 0.14 & 0.1130 & 54.4688 & 0.0783 & $0.559(11)$ \\
\hline $8432.26(08)$ & 54.6509 & 0.2067 & 0.38 & 0.2420 & 54.0891 & 0.2069 & $0.562(08)$ \\
\hline $8434.39(08)$ & 54.3352 & 0.0880 & 0.16 & 0.1231 & 53.7708 & 0.0882 & $0.564(06)$ \\
\hline $8436.34(08)$ & 54.2761 & 0.0850 & 0.16 & 0.1201 & 53.7094 & 0.0850 & $0.567(03)$ \\
\hline $8438.54(08)$ & 53.9127 & 0.0847 & 0.16 & 0.1196 & 53.3434 & 0.0847 & $0.569(01)$ \\
\hline $8440.61(08)$ & 53.7910 & 0.0895 & 0.17 & 0.1244 & 53.2192 & 0.0896 & $0.572(02)$ \\
\hline $8442.56(08)$ & 53.9991 & 0.0908 & 0.17 & 0.1258 & 53.4250 & 0.0909 & $0.574(04)$ \\
\hline $8444.65(08)$ & 54.2086 & 0.0798 & 0.15 & 0.1149 & 53.6321 & 0.0801 & $0.577(06)$ \\
\hline $8446.72(08)$ & 54.3395 & 0.0918 & 0.17 & 0.1270 & 53.7605 & 0.0923 & $0.579(09)$ \\
\hline 8448.62(08) & 54.3923 & 0.0799 & 0.15 & 0.1150 & 53.8120 & 0.0805 & $0.580(10)$ \\
\hline $8450.57(08)$ & 54.6064 & 0.0851 & 0.16 & 0.1204 & 54.0264 & 0.0857 & $0.580(10)$ \\
\hline $8452.59(08)$ & 54.8616 & 0.1052 & 0.19 & 0.1406 & 54.2818 & 0.1056 & $0.580(10)$ \\
\hline $8454.49(08)$ & 54.9462 & 0.1724 & 0.31 & 0.2079 & 54.3667 & 0.1727 & $0.580(09)$ \\
\hline $8456.39(08)$ & 54.5410 & 0.0880 & 0.16 & 0.1232 & 53.9618 & 0.0885 & $0.579(09)$ \\
\hline $8458.48(08)$ & 54.6889 & 0.0854 & 0.16 & 0.1207 & 54.1099 & 0.0858 & $0.579(09)$ \\
\hline $8460.38(08)$ & 54.7483 & 0.0945 & 0.17 & 0.1298 & 54.1696 & 0.0949 & $0.579(09)$ \\
\hline $8462.40(08)$ & 54.5447 & 0.0899 & 0.16 & 0.1252 & 53.9662 & 0.0903 & $0.579(08)$ \\
\hline $8464.49(08)$ & 54.8027 & 0.0854 & 0.16 & 0.1208 & 54.2245 & 0.0858 & $0.578(08)$ \\
\hline $8466.45(07)$ & 54.7076 & 0.0721 & 0.13 & 0.1074 & 54.1296 & 0.0725 & $0.578(08)$ \\
\hline $8468.85(08)$ & 54.7508 & 0.0916 & 0.17 & 0.1270 & 54.1731 & 0.0919 & $0.578(08)$ \\
\hline $8479.07(08)$ & 54.5002 & 0.0816 & 0.15 & 0.1169 & 53.9239 & 0.0819 & $0.576(06)$ \\
\hline $8488.88(08)$ & 54.4326 & 0.0819 & 0.15 & 0.1170 & 53.8575 & 0.0820 & $0.575(05)$ \\
\hline $8498.89(08)$ & 54.5378 & 0.1453 & 0.27 & 0.1806 & 53.9639 & 0.1454 & $0.574(04)$ \\
\hline $8509.12(08)$ & 52.9093 & 0.1244 & 0.24 & 0.1587 & 52.3359 & 0.1244 & $0.573(04)$ \\
\hline $8518.93(08)$ & 52.5364 & 0.0781 & 0.15 & 0.1122 & 51.9634 & 0.0782 & $0.573(04)$ \\
\hline $8528.96(08)$ & 51.8447 & 0.1144 & 0.22 & 0.1481 & 51.2721 & 0.1145 & $0.573(04)$ \\
\hline $8539.26(08)$ & 51.9105 & 0.0792 & 0.15 & 0.1129 & 51.3383 & 0.0793 & $0.572(04)$ \\
\hline $8548.96(08)$ & 51.3640 & 0.0799 & 0.16 & 0.1134 & 50.7922 & 0.0801 & $0.572(05)$ \\
\hline $8559.00(06)$ & 51.8442 & 0.0583 & 0.11 & 0.0920 & 51.2728 & 0.0585 & $0.571(05)$ \\
\hline $8569.31(08)$ & 51.0806 & 0.1135 & 0.22 & 0.1468 & 50.5096 & 0.1136 & $0.571(05)$ \\
\hline $8579.02(08)$ & 51.1984 & 0.0893 & 0.17 & 0.1226 & 50.6278 & 0.0894 & $0.571(05)$ \\
\hline 8589.07(08) & 50.4235 & 0.0918 & 0.18 & 0.1247 & 49.8533 & 0.0920 & $0.570(05)$ \\
\hline 8599.33(08) & 50.5602 & 0.0902 & 0.18 & 0.1232 & 49.9905 & 0.0904 & $0.570(05)$ \\
\hline $8609.30(06)$ & 50.0431 & 0.0840 & 0.17 & 0.1167 & 49.4738 & 0.0842 & $0.569(06)$ \\
\hline $8619.42(07)$ & 50.2759 & 0.0648 & 0.13 & 0.0976 & 49.7070 & 0.0650 & $0.569(06)$ \\
\hline 8639.67(13) & 48.9375 & 0.1421 & 0.29 & 0.1742 & 48.3695 & 0.1423 & $0.568(06)$ \\
\hline
\end{tabular}




\begin{tabular}{|c|c|c|c|c|c|c|c|}
\hline 8639.73(09) & 48.8728 & 0.1747 & 0.36 & 0.2067 & 48.3048 & 0.1748 & $0.568(06)$ \\
\hline 8659.63(08) & 48.8465 & 0.1009 & 0.21 & 0.1329 & 48.2793 & 0.1011 & $0.567(06)$ \\
\hline 8679.37(09) & 47.7008 & 0.1902 & 0.40 & 0.2216 & 47.1344 & 0.1903 & $0.566(07)$ \\
\hline 8679.43(13) & 47.3296 & 0.1504 & 0.32 & 0.1816 & 46.7633 & 0.1506 & $0.566(07)$ \\
\hline 8699.71(08) & 48.0933 & 0.0890 & 0.19 & 0.1205 & 47.5279 & 0.0892 & $0.565(07)$ \\
\hline 8719.44(13) & 47.7475 & 0.1449 & 0.30 & 0.1763 & 47.1829 & 0.1451 & $0.565(07)$ \\
\hline 8719.50(09) & 47.2965 & 0.0964 & 0.20 & 0.1275 & 46.7319 & 0.0967 & $0.565(07)$ \\
\hline $8739.52(07)$ & 47.6887 & 0.0833 & 0.17 & 0.1146 & 47.1250 & 0.0836 & $0.564(08)$ \\
\hline $8759.70(07)$ & 46.6689 & 0.0770 & 0.16 & 0.1078 & 46.1062 & 0.0774 & $0.563(08)$ \\
\hline 8779.37(07) & 47.0756 & 0.0775 & 0.16 & 0.1085 & 46.5138 & 0.0780 & $0.562(08)$ \\
\hline 8799.67(07) & 46.0529 & 0.0904 & 0.20 & 0.1208 & 45.4920 & 0.0908 & $0.561(09)$ \\
\hline $8819.54(07)$ & 46.1991 & 0.0774 & 0.17 & 0.1079 & 45.6392 & 0.0779 & $0.560(09)$ \\
\hline $8839.82(05)$ & 45.6728 & 0.1019 & 0.22 & 0.1321 & 45.1138 & 0.1023 & $0.559(09)$ \\
\hline $8870.07(05)$ & 46.0757 & 0.0560 & 0.12 & 0.0864 & 45.5182 & 0.0568 & $0.558(09)$ \\
\hline 8900.11(05) & 45.0012 & 0.1369 & 0.30 & 0.1667 & 44.4451 & 0.1372 & $0.556(10)$ \\
\hline $8930.22(05)$ & 45.5676 & 0.0544 & 0.12 & 0.0845 & 45.0130 & 0.0553 & $0.555(10)$ \\
\hline 8960.14(09) & 44.7945 & 0.1007 & 0.22 & 0.1304 & 44.2414 & 0.1012 & $0.553(11)$ \\
\hline $8960.21(06)$ & 44.3669 & 0.0903 & 0.20 & 0.1198 & 43.8138 & 0.0909 & $0.553(11)$ \\
\hline $8990.12(05)$ & 44.9562 & 0.0591 & 0.13 & 0.0889 & 44.4046 & 0.0601 & $0.552(11)$ \\
\hline $9020.24(07)$ & 44.1507 & 0.1894 & 0.43 & 0.2187 & 43.6004 & 0.1897 & $0.550(11)$ \\
\hline $9060.44(06)$ & 44.4859 & 0.0624 & 0.14 & 0.0919 & 43.9373 & 0.0634 & $0.549(11)$ \\
\hline $9100.87(07)$ & 42.6163 & 0.1483 & 0.35 & 0.1767 & 42.0694 & 0.1487 & $0.547(11)$ \\
\hline 9140.95(08) & 42.8422 & 0.0780 & 0.18 & 0.1066 & 42.2971 & 0.0788 & $0.545(11)$ \\
\hline 9181.03(08) & 41.6936 & 0.1522 & 0.37 & 0.1801 & 41.1502 & 0.1526 & $0.543(12)$ \\
\hline 9221.10(08) & 41.8914 & 0.0770 & 0.18 & 0.1050 & 41.3498 & 0.0779 & $0.542(12)$ \\
\hline $9261.02(10)$ & 41.0080 & 0.2142 & 0.52 & 0.2417 & 40.4682 & 0.2145 & $0.540(12)$ \\
\hline $9261.09(14)$ & 40.5252 & 0.1467 & 0.36 & 0.1740 & 39.9854 & 0.1472 & $0.540(12)$ \\
\hline 9301.07(10) & 41.5239 & 0.1076 & 0.26 & 0.1354 & 40.9859 & 0.1082 & $0.538(12)$ \\
\hline 9341.17(08) & 40.3366 & 0.1159 & 0.29 & 0.1431 & 39.8005 & 0.1166 & $0.536(12)$ \\
\hline 9380.95(08) & 40.7612 & 0.0887 & 0.22 & 0.1161 & 40.2269 & 0.0896 & $0.534(12)$ \\
\hline $9471.67(09)$ & 40.6914 & 0.0795 & 0.20 & 0.1068 & 40.1615 & 0.0804 & $0.530(12)$ \\
\hline 9521.81(09) & 40.2717 & 0.1644 & 0.41 & 0.1915 & 39.7442 & 0.1648 & $0.528(12)$ \\
\hline
\end{tabular}

